\title{
On Positive Solutions and Mann Iterative Schemes of a Third Order Difference Equation
}

\author{
Zeqing Liu, ${ }^{1}$ Heng Wu, ${ }^{1}$ Shin Min Kang, ${ }^{2}$ and Young Chel Kwun ${ }^{3}$ \\ ${ }^{1}$ Department of Mathematics, Liaoning Normal University, Dalian, Liaoning 116029, China \\ ${ }^{2}$ Department of Mathematics and RINS, Gyeongsang National University, Jinju 660-701, Republic of Korea \\ ${ }^{3}$ Department of Mathematics, Dong-A University, Pusan 614-714, Republic of Korea
}

Correspondence should be addressed to Young Chel Kwun; yckwun@dau.ac.kr

Received 14 October 2013; Accepted 16 December 2013; Published 28 January 2014

Academic Editor: Zhi-Bo Huang

Copyright (C) 2014 Zeqing Liu et al. This is an open access article distributed under the Creative Commons Attribution License, which permits unrestricted use, distribution, and reproduction in any medium, provided the original work is properly cited.

The existence of uncountably many positive solutions and convergence of the Mann iterative schemes for a third order nonlinear neutral delay difference equation are proved. Six examples are given to illustrate the results presented in this paper.

\section{Introduction and Preliminaries}

Recently, many researchers studied the oscillation, nonoscillation, and existence of solutions for linear and nonlinear second and third order difference equations and systems see, for example, [1-23] and the references cited therein. By means of the Reccati transformation techniques, Saker [18] discussed the third order difference equation

$$
\Delta^{3} x_{n}+p_{n} x_{n+1}=0, \quad \forall n \geq n_{0},
$$

and presented some sufficient conditions which ensure that all solutions are to be oscillatory or tend to zero. Utilizing the Schauder fixed point theorem, Yan and Liu [22] proved the existence of a bounded nonoscillatory solution for the third order difference equation

$$
\Delta^{3} x_{n}+f\left(n, x_{n}, x_{n-\tau}\right)=0, \quad \forall n \geq n_{0} .
$$

Agarwal [2] established the oscillatory and asymptotic properties for the third order nonlinear difference equation

$$
\Delta^{3} x_{n}+q_{n} f\left(x_{n+1}\right)=0, \quad \forall n \geq 1 .
$$

Andruch-Sobiło and Migda [4] studied the third order linear difference equation of neutral type

$$
\Delta^{3}\left(x_{n}-p_{n} x_{\sigma n}\right) \pm q_{n} x_{\tau n}=0, \quad \forall n \geq n_{0},
$$

and obtained sufficient conditions which ensure that all solutions of the equation are oscillatory. Grace and Hamedani [6] discussed the difference equation

$$
\Delta^{3}\left(x_{n}-x_{n-\tau}\right) \pm q_{n}\left|x_{n-\sigma}\right|^{3} \operatorname{sgn} x_{n-\sigma}=0, \quad \forall n \geq 0,
$$

and gave some new criteria for the oscillation of all solutions and all bounded solutions.

Our goal is to discuss solvability and convergence of the Mann iterative schemes for the following third order nonlinear neutral delay difference equation:

$$
\begin{aligned}
& \Delta^{3}\left(x_{n}+b_{n} x_{n-\tau}\right)+\Delta h\left(n, x_{h_{1 n}}, x_{h_{2 n}}, \ldots, x_{h_{k n}}\right) \\
& \quad+f\left(n, x_{f_{1 n}}, x_{f_{2 n}}, \ldots, x_{f_{k n}}\right)=c_{n}, \quad \forall n \geq n_{0},
\end{aligned}
$$

where $\tau, k, n_{0} \in \mathbb{N},\left\{b_{n}\right\}_{n \in \mathbb{N}_{n_{0}}},\left\{c_{n}\right\}_{n \in \mathbb{N}_{n_{0}}} \subset \mathbb{R}, h, f \in C\left(\mathbb{N}_{n_{0}} \times\right.$ $\left.\mathbb{R}^{k}, \mathbb{R}\right),\left\{h_{l n}\right\}_{n \in \mathbb{N}_{n_{0}}},\left\{f_{l n}\right\}_{n \in \mathbb{N}_{n_{0}}} \subseteq \mathbb{N}$, and

$$
\lim _{n \rightarrow \infty} h_{l n}=\lim _{n \rightarrow \infty} f_{l n}=+\infty, \quad l \in\{1,2, \ldots, k\} .
$$

By employing the Banach fixed point theorem and some new techniques, we establish the existence of uncountably many positive solutions of (6), conceive a few Mann iterative schemes for approximating these positive solutions, and prove their convergence and the error estimates. Six nontrivial examples are included. 
Throughout this paper, we assume that $\Delta$ is the forward difference operator defined by $\Delta x_{n}=x_{n+1}-x_{n}, \mathbb{R}=$ $(-\infty,+\infty), \mathbb{R}^{+}=[0,+\infty) \mathbb{N}_{0}$ and $\mathbb{N}$ denote the sets of nonnegative integers and positive integers, respectively,

$$
\begin{aligned}
& \mathbb{N}_{t}=\{n: n \in \mathbb{N} \text { with } n \geq t\}, \quad \forall t \in \mathbb{N}, \\
& \beta=\min \left\{n_{0}-\tau, \inf \left\{h_{l n}, f_{l n}: 1 \leq l \leq k, n \in \mathbb{N}_{n_{0}}\right\}\right\} \in \mathbb{N}, \\
& H_{n}=\max \left\{h_{l n}^{2}: l \in\{1,2, \ldots, k\}\right\}, \quad \forall n \in \mathbb{N}_{n_{0}}, \\
& F_{n}=\max \left\{f_{l n}^{2}: l \in\{1,2, \ldots, k\}\right\}, \quad \forall n \in \mathbb{N}_{n_{0}},
\end{aligned}
$$

and $l_{\beta}^{\infty}$ represents the Banach space of all real sequences on $\mathbb{N}_{\beta}$ with norm

$$
\begin{array}{r}
\|x\|=\sup _{n \in \mathbb{N}_{\beta}}\left|\frac{x_{n}}{n^{2}}\right|<+\infty \quad \text { for each } x=\left\{x_{n}\right\}_{n \in \mathbb{N}_{\beta}} \in l_{\beta}^{\infty}, \\
A(N, M)=\left\{x=\left\{x_{n}\right\}_{n \in \mathbb{N}_{\beta}} \in l_{\beta}^{\infty}: N \leq \frac{x_{n}}{n^{2}} \leq M, n \in \mathbb{N}_{\beta}\right\} \\
\text { for any } M>N>0 .
\end{array}
$$

It is easy to see that $A(N, M)$ is a closed and convex subset of $l_{\beta}^{\infty}$. By a solution of (6), we mean a sequence $\left\{x_{n}\right\}_{n \in \mathbb{N}_{\beta}}$ with a positive integer $T \geq n_{0}+\tau+\beta$ such that (6) holds for all $n \geq$ $T$.

Lemma 1. Let $\left\{p_{t}\right\}_{t \in \mathbb{N}}$ be a nonnegative sequence and $\tau \in \mathbb{N}$.

(i) If $\lim _{n \rightarrow \infty}\left(1 / n^{2}\right) \sum_{t=n+\tau}^{\infty} t^{2} p_{t} \quad=\quad 0$, then $\lim _{n \rightarrow \infty}\left(1 / n^{2}\right) \sum_{i=1}^{\infty} \sum_{s=n+i \tau}^{\infty} \sum_{t=s}^{\infty} p_{t}=0$.

(ii) If $\lim _{n \rightarrow \infty}\left(1 / n^{2}\right) \sum_{t=n+\tau}^{\infty} t^{3} p_{t} \quad=\quad 0$, then $\lim _{n \rightarrow \infty}\left(1 / n^{2}\right) \sum_{i=1}^{\infty} \sum_{u=n+i \tau}^{\infty} \sum_{s=u}^{\infty} \sum_{t=s}^{\infty} p_{t}=0$.

Proof. Note that

$$
\begin{aligned}
0 & \leq \frac{1}{n^{2}} \sum_{i=1}^{\infty} \sum_{s=n+i \tau}^{\infty} \sum_{t=s}^{\infty} p_{t} \\
& =\frac{1}{n^{2}} \sum_{i=1}^{\infty}\left(\sum_{t=n+i \tau}^{\infty} p_{t}+\sum_{t=n+1+i \tau}^{\infty} p_{t}+\sum_{t=n+2+i \tau}^{\infty} p_{t}+\cdots\right) \\
& =\frac{1}{n^{2}} \sum_{i=1}^{\infty} \sum_{t=n+i \tau}^{\infty}(1+t-n-i \tau) p_{t} \leq \frac{1}{n^{2}} \sum_{i=1}^{\infty} \sum_{t=n+i \tau}^{\infty} t p_{t} \\
& =\frac{1}{n^{2}}\left(\sum_{t=n+\tau}^{\infty} t p_{t}+\sum_{t=n+2 \tau}^{\infty} t p_{t}+\sum_{t=n+3 \tau}^{\infty} t p_{t}+\cdots\right) \\
& \leq \frac{1}{n^{2}} \sum_{t=n+\tau}^{\infty}\left(1+\frac{t-n-\tau}{\tau}\right) t p_{t}=\frac{1}{n^{2}} \sum_{t=n+\tau}^{\infty} \frac{t-n}{\tau} t p_{t} \\
& \leq \frac{1}{n^{2} \tau} \sum_{t=n+\tau}^{\infty} t^{2} p_{t} \longrightarrow 0 \text { as } n \longrightarrow \infty
\end{aligned}
$$

that is,

$$
\lim _{n \rightarrow \infty} \frac{1}{n^{2}} \sum_{i=1}^{\infty} \sum_{s=n+i \tau}^{\infty} \sum_{t=s}^{\infty} p_{t}=0 .
$$

As in the proof of (10), we infer that

$$
\begin{aligned}
0 & \leq \frac{1}{n^{2}} \sum_{i=1}^{\infty} \sum_{u=n+i \tau}^{\infty} \sum_{s=u}^{\infty} \sum_{t=s}^{\infty} p_{t} \\
& =\frac{1}{n^{2}} \sum_{i=1}^{\infty} \sum_{u=n+i \tau}^{\infty} \sum_{t=u}^{\infty}(1+t-u) p_{t} \\
& \leq \frac{1}{n^{2}} \sum_{i=1}^{\infty} \sum_{u=n+i \tau}^{\infty} \sum_{t=u}^{\infty} t p_{t} \leq \frac{1}{n^{2} \tau} \sum_{t=n+\tau}^{\infty} t^{3} p_{t} \longrightarrow 0 \quad \text { as } n \longrightarrow \infty,
\end{aligned}
$$

which implies that

$$
\lim _{n \rightarrow \infty} \frac{1}{n^{2}} \sum_{i=1}^{\infty} \sum_{u=n+i \tau}^{\infty} \sum_{s=u}^{\infty} \sum_{t=s}^{\infty} p_{t}=0 .
$$

This completes the proof.

\section{Uncountably Many Positive Solutions and Mann Iterative Schemes}

In this section, using the Banach fixed point theorem and Mann iterative schemes, we establish the existence of uncountably many positive solutions of (6), prove convergence of the Mann iterative schemes relative to these positive solutions, and compute the error estimates between the Mann iterative schemes and the positive solutions.

Theorem 2. Assume that there exist two constants $M$ and $N$ with $M>N>0$ and four nonnegative sequences $\left\{P_{n}\right\}_{n \in \mathbb{N}_{n_{0}}}, \quad\left\{Q_{n}\right\}_{n \in \mathbb{N}_{n_{0}}}, \quad\left\{R_{n}\right\}_{n \in \mathbb{N}_{n_{0}}}$ and $\left\{W_{n}\right\}_{n \in \mathbb{N}_{n_{0}}}$ satisfying

$$
\begin{gathered}
\left|f\left(n, u_{1}, u_{2}, \ldots, u_{k}\right)-f\left(n, \bar{u}_{1}, \bar{u}_{2}, \ldots, \bar{u}_{k}\right)\right| \\
\leq P_{n} \max \left\{\left|u_{l}-\bar{u}_{l}\right|: 1 \leq l \leq k\right\}, \\
\left|h\left(n, u_{1}, u_{2}, \ldots, u_{k}\right)-h\left(n, \bar{u}_{1}, \bar{u}_{2}, \ldots, \bar{u}_{k}\right)\right| \\
\leq R_{n} \max \left\{\left|u_{l}-\bar{u}_{l}\right|: 1 \leq l \leq k\right\}, \\
\forall\left(n, u_{l}, \bar{u}_{l}\right) \in \mathbb{N}_{n_{0}} \times\left(\mathbb{R}^{+} \backslash\{0\}\right)^{2}, \quad 1 \leq l \leq k ; \\
\left|f\left(n, u_{1}, u_{2}, \ldots, u_{k}\right)\right| \leq Q_{n}, \quad\left|h\left(n, u_{1}, u_{2}, \ldots, u_{k}\right)\right| \leq W_{n}, \\
\forall\left(n, u_{l}\right) \in \mathbb{N}_{n_{0}} \times\left(\mathbb{R}^{+} \backslash\{0\}\right), \quad 1 \leq l \leq k ; \\
\lim _{n \rightarrow \infty} \frac{1}{n^{2}} \sum_{i=1}^{\infty} \sum_{u=n+i \tau}^{\infty} \sum_{s=u}^{\infty} \max \left\{R_{s} H_{s}, W_{s}\right\}=0 ; \\
\lim _{n \rightarrow \infty} \frac{1}{n^{2}} \sum_{i=1}^{\infty} \sum_{u=n+i \tau}^{\infty} \sum_{s=u}^{\infty} \sum_{t=s}^{\infty} \max \left\{P_{t} F_{t}, Q_{t},\left|c_{t}\right|\right\}=0 ; \\
b_{n}=-1 \text { eventually. }
\end{gathered}
$$


Then one has the following.

(a) For any $L \in(N, M)$, there exist $\theta \in(0,1)$ and $T \geq$ $n_{0}+\tau+\beta$ such that, for each $x_{0}=\left\{x_{0 n}\right\}_{n \in \mathbb{N}_{\beta}} \in$ $A(N, M)$, the Mann iterative sequence $\left\{x_{m}\right\}_{m \in \mathbb{N}_{0}}=$ $\left\{\left\{x_{m n}\right\}_{n \in \mathbb{N}_{\beta}}\right\}_{m \in \mathbb{N}_{0}}$ generated by the scheme

$x_{m+1 n}$

$$
\begin{aligned}
& \left\{\begin{array}{l}
\left(1-\alpha_{m}\right) x_{m n} \\
+\alpha_{m}\left\{n^{2} L\right. \\
\quad+\sum_{i=1}^{\infty} \sum_{u=n+i \tau}^{\infty} \sum_{s=u}^{\infty}\left[h\left(s, x_{m h_{1 s}}, x_{m h_{2 s}}, \ldots, x_{m h_{k s}}\right)\right.
\end{array}\right. \\
& -\sum_{t=s}^{\infty}\left(f\left(t, x_{m f_{1 t}}, x_{m f_{2 t}}, \ldots, x_{m f_{k t}}\right)\right. \\
& \left.\left.\left.-c_{t}\right)\right]\right\} \text {, } \\
& =\left\{\begin{array}{c}
n \geq T, \quad m \geq 0, \\
\left(1-\alpha_{m}\right) x_{m T} \\
+\alpha_{m}\left\{T^{2} L\right. \\
\quad+\sum_{i=1}^{\infty} \sum_{u=T+i \tau}^{\infty} \sum_{s=u}^{\infty}\left[h\left(s, x_{m h_{1 s}}, x_{m h_{2 s}}, \ldots, x_{m h_{k s}}\right)\right.
\end{array}\right. \\
& -\sum_{t=s}^{\infty}\left(f\left(t, x_{m f_{1 t}}, x_{m f_{2 t}}, \ldots, x_{m f_{k t}}\right)\right. \\
& \left.\left.\left.-c_{t}\right)\right]\right\} \text {, } \\
& \beta \leq n<T, \quad m \geq 0 \text {, }
\end{aligned}
$$

converges to a positive solution $z=\left\{z_{n}\right\}_{n \in \mathbb{N}_{\beta}} \in A(N, M)$ of (6) with $\lim _{n \rightarrow \infty} z_{n}=+\infty$ and has the following error estimate:

$$
\left\|x_{m+1}-z\right\| \leq e^{-(1-\theta) \sum_{i=0}^{m} \alpha_{i}}\left\|x_{m}-z\right\|, \quad \forall m \in \mathbb{N}_{0},
$$

where $\left\{\alpha_{m}\right\}_{m \in \mathbb{N}_{0}}$ is an arbitrary sequence in $[0,1]$ such that

$$
\sum_{m=0}^{\infty} \alpha_{m}=+\infty
$$

(b) Equation (6) possesses uncountably many positive solutions in $A(N, M)$.

Proof. Firstly, we show that (a) holds. Put $L \in(N, M)$. It follows from (16) (18) that there exist $\theta \in(0,1)$ and $T \geq$ $n_{0}+\tau+\beta$ satisfying

$$
\begin{gathered}
\theta=\frac{1}{T^{2}} \sum_{i=1}^{\infty} \sum_{u=T+i \tau}^{\infty} \sum_{s=u}^{\infty}\left(R_{s} H_{s}+\sum_{t=s}^{\infty} P_{t} F_{t}\right) ; \\
\frac{1}{T^{2}} \sum_{i=1}^{\infty} \sum_{u=T+i \tau}^{\infty} \sum_{s=u}^{\infty}\left(W_{s}+\sum_{t=s}^{\infty}\left(Q_{t}+\left|c_{t}\right|\right)\right) \\
<\min \{M-L, L-N\} ; \\
b_{n}=-1, \quad \forall n \geq T .
\end{gathered}
$$

Define a mapping $S_{L}: A(N, M) \rightarrow l_{\beta}^{\infty}$ by

$$
\begin{aligned}
& S_{L} x_{n} \\
& =\left\{\begin{array}{r}
n^{2} L \\
+\sum_{i=1}^{\infty} \sum_{u=n+i \tau}^{\infty} \sum_{s=u}^{\infty}\left\{h\left(s, x_{h_{1 s}}, x_{h_{2 s}}, \ldots, x_{h_{k s}}\right)\right. \\
\left.-\sum_{t=s}^{\infty}\left[f\left(t, x_{f_{1 t}}, x_{f_{2 t}}, \ldots, x_{f_{k t}}\right)-c_{t}\right]\right\}, \\
n \geq T, \quad S_{L} x_{T}, \quad \beta \leq n<T,
\end{array}\right.
\end{aligned}
$$

for each $x=\left\{x_{n}\right\}_{n \in \mathbb{N}_{\beta}} \in A(N, M)$. In light of (14), (15), (22), (23), and (25), we obtain that for each $x=\left\{x_{n}\right\}_{n \in \mathbb{N}_{\beta}}, y=$ $\left\{y_{n}\right\}_{n \in \mathbb{N}_{\beta}} \in A(N, M)$

$$
\begin{aligned}
&\left|\frac{S_{L} x_{n}}{n^{2}}-\frac{S_{L} y_{n}}{n^{2}}\right| \\
& \leq \frac{1}{n^{2}} \sum_{i=1}^{\infty} \sum_{u=n+i \tau}^{\infty} \sum_{s=u}^{\infty}[\mid h\left(s, x_{h_{1 s}}, x_{h_{2 s}}, \ldots, x_{h_{k s}}\right) \\
&-h\left(s, y_{h_{1 s}}, y_{h_{2 s}}, \ldots, y_{h_{k s}}\right) \mid \\
&+ \sum_{t=s}^{\infty} \mid f\left(t, x_{f_{1 t}}, x_{f_{2 t}}, \ldots, x_{f_{k t}}\right) \\
&\left.-f\left(t, y_{f_{1 t}}, y_{f_{2 t}}, \ldots, y_{f_{k t}}\right) \mid\right]
\end{aligned}
$$$$
\leq \frac{1}{n^{2}} \sum_{i=1}^{\infty} \sum_{u=n+i \tau}^{\infty} \sum_{s=u}^{\infty}\left[R_{s} \max \left\{\left|x_{h_{l s}}-y_{h_{l s}}\right|: 1 \leq l \leq k\right\}\right.
$$$$
\left.+\sum_{t=s}^{\infty} P_{t} \max \left\{\left|x_{f_{l t}}-y_{f_{l t}}\right|: 1 \leq l \leq k\right\}\right]
$$$$
\leq \frac{\|x-y\|}{n^{2}} \sum_{i=1}^{\infty} \sum_{u=n+i \tau}^{\infty} \sum_{s=u}^{\infty}\left[R_{s} \max \left\{h_{l s}^{2}: 1 \leq l \leq k\right\}\right.
$$$$
\left.+\sum_{t=s}^{\infty} P_{t} \max \left\{f_{l t}^{2}: 1 \leq l \leq k\right\}\right]
$$$$
\leq \frac{\|x-y\|}{T^{2}} \sum_{i=1}^{\infty} \sum_{u=T+i \tau}^{\infty} \sum_{s=u}^{\infty}\left(R_{s} H_{s}+\sum_{t=s}^{\infty} P_{t} F_{t}\right)
$$$$
=\theta\|x-y\|,
$$$$
\left|\frac{S_{L} x_{n}}{n^{2}}-L\right|
$$$$
=\mid \frac{1}{n^{2}} \sum_{i=1}^{\infty} \sum_{u=n+i \tau}^{\infty} \sum_{s=u}^{\infty}\left\{h\left(s, x_{h_{1 s}}, x_{h_{2 s}}, \ldots, x_{h_{k s}}\right)\right.
$$$$
\left.-\sum_{t=s}^{\infty}\left[f\left(t, x_{f_{1 t}}, x_{f_{2 t}}, \ldots, x_{f_{k t}}\right)-c_{t}\right]\right\} \mid
$$

$\leq \frac{1}{n^{2}} \sum_{i=1}^{\infty} \sum_{u=n+i \tau}^{\infty} \sum_{s=u}^{\infty}\left\{\left|h\left(s, x_{h_{1 s}}, x_{h_{2 s}}, \ldots, x_{h_{k s}}\right)\right|\right.$ 


$$
\begin{aligned}
& \left.+\sum_{t=s}^{\infty}\left[\left|f\left(t, x_{f_{1 t}}, x_{f_{2 t}}, \ldots, x_{f_{k t}}\right)\right|+\left|c_{t}\right|\right]\right\} \\
& \leq \frac{1}{T^{2}} \sum_{i=1}^{\infty} \sum_{u=T+i \tau}^{\infty} \sum_{s=u}^{\infty}\left[W_{s}+\sum_{t=s}^{\infty}\left(Q_{t}+\left|c_{t}\right|\right)\right] \\
& <\min \{M-L, L-N\},
\end{aligned}
$$

which yield that

$$
S_{L}(A(N, M)) \subseteq A(N, M),
$$

$$
\left\|S_{L} x-S_{L} y\right\| \leq \theta\|x-y\|, \quad \forall x, y \in A(N, M),
$$

which implies that $S_{L}$ is a contraction in $A(N, M)$. The Banach fixed point theorem and (27) ensure that $S_{L}$ has a unique fixed point $z=\left\{z_{n}\right\}_{n \in \mathbb{N}_{\beta}} \in A(N, M)$; that is,

$$
\begin{aligned}
& z_{n}= n^{2} L \\
&+\sum_{i=1}^{\infty} \sum_{u=n+i \tau}^{\infty} \sum_{s=u}^{\infty}\left\{h\left(s, z_{h_{1 s}}, z_{h_{2 s}}, \ldots, z_{h_{k s}}\right)\right. \\
&\left.-\sum_{t=s}^{\infty}\left[f\left(t, z_{f_{1 t}}, z_{f_{2 t}}, \ldots, z_{f_{k t}}\right)-c_{t}\right]\right\}, \\
& z_{n-\tau}=(n-\tau)^{2} L \quad \forall n \geq T, \\
&+\sum_{i=1}^{\infty} \sum_{u=n+(i-1) \tau}^{\infty} \sum_{s=u}^{\infty}\left\{h\left(s, z_{h_{1 s}}, z_{h_{2 s}}, \ldots, z_{h_{k s}}\right)\right. \\
&-\sum_{t=s}^{\infty}\left[f\left(t, z_{f_{1 t}}, z_{f_{2 t}}, \ldots, z_{f_{k t}}\right)\right. \\
&\left.\left.-c_{t}\right]\right\}, \quad \forall n \geq T+\tau,
\end{aligned}
$$

which mean that

$$
\begin{aligned}
z_{n}-z_{n-\tau}=\left(2 n \tau-\tau^{2}\right) L & \\
-\sum_{u=n} \sum_{s=u}^{\infty}\left\{h\left(s, z_{h_{1 s}}, z_{h_{2 s}}, \ldots, z_{h_{k s}}\right)\right. & \\
& \left.-\sum_{t=s}^{\infty}\left[f\left(t, z_{f_{1 t}}, z_{f_{2 t}}, \ldots, z_{f_{k t}}\right)-c_{t}\right]\right\}, \\
\forall n \geq T+\tau, & \forall n \geq
\end{aligned}
$$

which yields that

$$
\begin{aligned}
& \Delta\left(z_{n}-z_{n-\tau}\right) \\
& =2 \tau L+\sum_{s=n}^{\infty}\left\{h\left(s, z_{h_{1 s}}, z_{h_{2 s}}, \ldots, z_{h_{k s}}\right)\right. \\
& \left.-\sum_{t=s}^{\infty}\left[f\left(t, z_{f_{1 t}}, z_{f_{2 t}}, \ldots, z_{f_{k t}}\right)-c_{t}\right]\right\}, \\
& \forall n \geq T+\tau,
\end{aligned}
$$

$$
\begin{aligned}
\Delta^{2}\left(z_{n}-\right. & \left.z_{n-\tau}\right) \\
= & -h\left(n, z_{h_{1 n}}, z_{h_{2 n}}, \ldots, z_{h_{k n}}\right) \\
& +\sum_{t=n}^{\infty}\left[f\left(t, z_{f_{1 t}}, z_{f_{2 t}}, \ldots, z_{f_{k t}}\right)-c_{t}\right], \quad \forall n \geq T+\tau,
\end{aligned}
$$

which gives that

$$
\begin{aligned}
\Delta^{3}\left(z_{n}-\right. & \left.z_{n-\tau}\right) \\
= & -\Delta h\left(n, z_{h_{1 n}}, z_{h_{2 n}}, \ldots, z_{h_{k n}}\right) \\
& -f\left(t, z_{f_{1 n}}, z_{f_{2 n}}, \ldots, z_{f_{k n}}\right)+c_{n}, \quad \forall n \geq T+\tau,
\end{aligned}
$$

which together with (24) implies that $z=\left\{z_{n}\right\}_{n \in \mathbb{N}_{\beta}}$ is a positive solution of $(6)$ in $A(N, M)$. Note that

$$
N \leq \frac{z_{n}}{n^{2}} \leq M, \quad \forall n \in \mathbb{N}_{\beta}
$$

which guarantees that $\lim _{n \rightarrow \infty} z_{n}=+\infty$. It follows from (19), (22), (24), (25), and (27) that for any $m \in \mathbb{N}_{0}$ and $n \geq T$

$\left|\frac{x_{m+1 n}}{n^{2}}-\frac{z_{n}}{n^{2}}\right|$

$$
\begin{aligned}
& =\frac{1}{n^{2}} \mid\left(1-\alpha_{m}\right) x_{m n} \\
& +\alpha_{m}\left\{n^{2} L\right. \\
& +\sum_{i=1}^{\infty} \sum_{u=n+i \tau}^{\infty} \sum_{s=u}^{\infty}\left[h\left(s, x_{m h_{1 s}}, x_{m h_{2 s}}, \ldots, x_{m h_{k s}}\right)\right. \\
& -\sum_{t=s}^{\infty}\left(f \left(t, x_{m f_{1 t}}, x_{m f_{2 t}}, \ldots,\right.\right. \\
& \left.\left.\left.\left.x_{m f_{k t}}\right)-c_{t}\right)\right]\right\}-z_{n} \\
& \leq\left(1-\alpha_{m}\right) \frac{\left|x_{m n}-z_{n}\right|}{n^{2}}+\alpha_{m} \frac{\left|S_{L} x_{m n}-S_{L} z_{n}\right|}{n^{2}} \\
& \leq\left(1-\alpha_{m}\right)\left\|x_{m}-z\right\|+\theta \alpha_{m}\left\|x_{m}-z\right\| \\
& \leq\left[1-(1-\theta) \alpha_{m}\right]\left\|x_{m}-z\right\|, \quad \forall m \in \mathbb{N}_{0}, n \geq T,
\end{aligned}
$$

which implies that

$$
\left\|x_{m+1}-z\right\| \leq e^{-(1-\theta) \sum_{i=0}^{m} \alpha_{i}}\left\|x_{m}-z\right\|, \quad \forall m \in \mathbb{N}_{0} .
$$

That is, (20) holds. Thus Lemma 1, (20), and (21) guarantee that $\lim _{m \rightarrow \infty} x_{m}=z$.

Next we show that (b) holds. Let $L_{1}, L_{2} \in$ $(N, M)$ and $L_{1} \neq L_{2}$. As in the proof of (a), we deduce similarly that, for each $c \in\{1,2\}$, there exist constants $\theta_{c} \in$ $(0,1)$ and $T_{c} \geq n_{0}+\tau+\beta$ and a mapping $S_{L_{c}}$ satisfying 
(22) (27), where $\theta, L$, and $T$ are replaced by $\theta_{c}, L_{c}$, and $T_{c}$, respectively, and the mapping $S_{L_{c}}$ has a fixed point $z^{c}=$ $\left\{z_{n}^{c}\right\}_{n \in \mathbb{N}_{\beta}} \in A(N, M)$, which is a positive solution of (6) in $A(N, M)$ with $\lim _{n \rightarrow \infty} z_{n}^{c}=+\infty$. It follows that

$$
\begin{aligned}
& z_{n}^{c}=n^{2} L_{c} \\
&+\sum_{i=1}^{\infty} \sum_{u=n+i \tau}^{\infty} \sum_{s=u}^{\infty}\left\{h\left(s, z_{h_{1 s}}^{c}, z_{h_{2 s}}^{c}, \ldots, z_{h_{k s}}^{c}\right)\right. \\
&\left.-\sum_{t=s}^{\infty}\left[f\left(t, z_{f_{1 t}}^{c}, z_{f_{2 t}}^{c}, \ldots, z_{f_{k t}}^{c}\right)-c_{t}\right]\right\}, \\
& \forall n \geq T_{c},
\end{aligned}
$$

which together with (14) and (20) means that for $n \geq$ $\max \left\{T_{1}, T_{2}\right\}$

$$
\begin{aligned}
& \left|\frac{z_{n}^{1}}{n^{2}}-\frac{z_{n}^{2}}{n^{2}}\right| \\
& \geq\left|L_{1}-L_{2}\right| \\
& -\frac{1}{n^{2}} \sum_{i=1}^{\infty} \sum_{u=n+i \tau}^{\infty} \sum_{s=u}^{\infty} \mid h\left(s, z_{h_{1 s}}^{1}, z_{h_{2 s}}^{1}, \ldots, z_{h_{k s}}^{1}\right) \\
& -h\left(s, z_{h_{1 s}}^{2}, z_{h_{2 s}}^{2}, \ldots, z_{h_{k s}}^{2}\right) \\
& +\sum_{t=s}^{\infty}\left|f\left(t, z_{f_{1 t}}^{1}, z_{f_{2 t}}^{1}, \ldots, z_{f_{k t}}^{1}\right)-f\left(t, z_{f_{1 t}}^{2}, z_{f_{2 t}}^{2}, \ldots, z_{f_{k t}}^{2}\right)\right| \\
& \geq\left|L_{1}-L_{2}\right| \\
& -\frac{1}{n^{2}} \sum_{i=1}^{\infty} \sum_{u=n+i \tau}^{\infty} \sum_{s=u}^{\infty}\left[R_{s} \max \left\{\left|z_{h_{l s}}^{1}-z_{h_{l s}}^{2}\right|: 1 \leq l \leq k\right\}\right. \\
& \left.+\sum_{t=s}^{\infty} P_{t} \max \left\{\left|z_{f_{l t}}^{1}-z_{f_{l t}}^{2}\right|: 1 \leq l \leq k\right\}\right] \\
& \geq\left|L_{1}-L_{2}\right| \\
& -\frac{\left\|z^{1}-z^{2}\right\|}{n^{2}} \sum_{i=1}^{\infty} \sum_{u=n+i \tau}^{\infty} \sum_{s=u}^{\infty}\left(R_{s} H_{s}+\sum_{t=s}^{\infty} P_{t} F_{t}\right) \\
& \geq\left|L_{1}-L_{2}\right| \\
& -\frac{\left\|z^{1}-z^{2}\right\|}{\max \left\{T_{1}^{2}, T_{2}^{2}\right\}} \sum_{i=1}^{\infty} \sum_{u=\max \left\{T_{1}, T_{2}\right\}+i \tau}^{\infty} \sum_{s=u}^{\infty}\left(R_{s} H_{s}+\sum_{t=s}^{\infty} P_{t} F_{t}\right) \\
& \geq\left|L_{1}-L_{2}\right|-\max \left\{\theta_{1}, \theta_{2}\right\}\left\|z^{1}-z^{2}\right\| \text {, }
\end{aligned}
$$

which yields that

$$
\left\|z^{1}-z^{2}\right\| \geq \frac{\left|L_{1}-L_{2}\right|}{1+\max \left\{\theta_{1}, \theta_{2}\right\}}>0 ;
$$

that is, $z^{1} \neq z^{2}$. This completes the proof.
Theorem 3. Assume that there exist two constants $M$ and $N$ with $M>N>0$ and four nonnegative sequences $\left\{P_{n}\right\}_{n \in \mathbb{N}_{n}}$, $\left\{Q_{n}\right\}_{n \in \mathbb{N}_{n_{0}}},\left\{R_{n}\right\}_{n \in \mathbb{N}_{n_{0}}}$, and $\left\{W_{n}\right\}_{n \in \mathbb{N}_{n_{0}}}$ satisfying (14), (15), and

$$
\begin{gathered}
\lim _{n \rightarrow \infty} \frac{1}{n^{2}} \sum_{u=n}^{\infty} \sum_{s=u}^{\infty} \max \left\{R_{s} H_{s}, W_{s}\right\}=0 ; \\
\lim _{n \rightarrow \infty} \frac{1}{n^{2}} \sum_{u=n}^{\infty} \sum_{s=u}^{\infty} \sum_{t=s}^{\infty} \max \left\{P_{t} F_{t}, Q_{t},\left|c_{t}\right|\right\}=0 \\
b_{n}=1 \text { eventually. }
\end{gathered}
$$

Then one has the following.

(a) For any $L \in(N, M)$, there exist $\theta \in(0,1)$ and $T \geq$ $n_{0}+\tau+\beta$ such that, for each $x_{0}=\left\{x_{0 n}\right\}_{n \in \mathbb{N}_{\beta}} \in$ $A(N, M)$, the Mann iterative sequence $\left\{x_{m}\right\}_{m \in \mathbb{N}_{0}}=$ $\left\{\left\{x_{m n}\right\}_{n \in \mathbb{N}_{\beta}}\right\}_{m \in \mathbb{N}_{0}}$ generated by the scheme

$$
\begin{aligned}
& x_{m+1 n} \\
& \left\{\begin{array}{l}
\left(1-\alpha_{m}\right) x_{m n} \\
+\alpha_{m}\left\{n^{2} L\right. \\
\quad-\sum_{i=1}^{\infty} \sum_{u=n+(2 i-1) \tau}^{n+2 i \tau-1} \sum_{s=u}^{\infty}\left[h\left(s, x_{m h_{1 s}}, x_{m h_{2 s}}, \ldots, x_{m h_{k s}}\right)\right.
\end{array}\right. \\
& -\sum_{t=s}^{\infty}\left(f \left(t, x_{m f_{1 t}}, x_{m f_{2 t}}, \ldots,\right.\right. \\
& \left.\left.\left.\left.x_{m f_{k t}}\right)-c_{t}\right)\right]\right\} \text {, } \\
& n \geq T, \quad m \geq 0 \text {, } \\
& =\left\{\begin{array}{l}
\left(1-\alpha_{m}\right) x_{m T} \\
+\alpha_{m}\left\{T^{2} L\right.
\end{array}\right. \\
& +\alpha_{m}\left\{T^{2} L\right. \\
& -\sum_{i=1}^{\infty} \sum_{u=T+(2 i-1) \tau}^{T+2 i \tau-1} \sum_{s=u}^{\infty}\left[h\left(s, x_{m h_{1 s}}, x_{m h_{2 s}}, \ldots, x_{m h_{k s}}\right)\right. \\
& -\sum_{t=s}^{\infty}\left(f \left(t, x_{m f_{1 t}}, x_{m f_{2 t}}, \ldots,\right.\right. \\
& \left.\left.\left.\left.x_{m f_{k t}}\right)-c_{t}\right)\right]\right\}, \\
& \beta \leq n<T, \quad m \geq 0 \text {, }
\end{aligned}
$$

converges to a positive solution $z=\left\{z_{n}\right\}_{n \in \mathbb{N}_{\beta}} \in$ $A(N, M)$ of (6) with $\lim _{n \rightarrow \infty} z_{n}=+\infty$ and has the error estimate (20), where $\left\{\alpha_{m}\right\}_{m \in \mathbb{N}_{0}}$ is an arbitrary sequence in $[0,1]$ satisfying $(21)$.

(b) Equation (6) possesses uncountably many positive solutions in $A(N, M)$. 
Proof. Let $L \in(N, M)$. It follows from (38) (40) that there exist $\theta \in(0,1)$ and $T \geq n_{0}+\tau+\beta$ satisfying

$$
\begin{gathered}
\theta=\frac{1}{T^{2}} \sum_{u=T}^{\infty} \sum_{s=u}^{\infty}\left(R_{s} H_{s}+\sum_{t=s}^{\infty} P_{t} F_{t}\right) \\
\frac{1}{T^{2}} \sum_{u=T}^{\infty} \sum_{s=u}^{\infty}\left(W_{s}+\sum_{t=s}^{\infty}\left(Q_{t}+\left|c_{t}\right|\right)\right)<\min \{M-L, L-N\} \\
b_{n}=1, \quad \forall n \geq T .
\end{gathered}
$$

Define a mapping $S_{L}: A(N, M) \rightarrow l_{\beta}^{\infty}$ by

$$
\begin{aligned}
& S_{L} x_{n} \\
& =\left\{\begin{array}{rr}
n^{2} L \\
-\sum_{i=1}^{\infty} \sum_{u=n+(2 i-1) \tau}^{n+2 i \tau-1} \sum_{s=u}^{\infty}\left\{h\left(s, x_{h_{1 s}}, x_{h_{2 s}}, \ldots, x_{h_{k s}}\right)\right. \\
-\sum_{t=s}^{\infty}\left[f\left(t, x_{f_{1 t}}, x_{f_{2 t}}, \ldots, x_{f_{k t}}\right)\right. \\
\left.\left.-c_{t}\right]\right\}, \quad n \geq T, \\
\beta \leq n<T,
\end{array}\right.
\end{aligned}
$$

for each $x=\left\{x_{n}\right\}_{n \in \mathbb{N}_{\beta}} \in A(N, M)$. Using (14), (15), (42), (43), and (45), we get that for each $x=\left\{x_{n}\right\}_{n \in \mathbb{N}_{\beta}}, y=\left\{y_{n}\right\}_{n \in \mathbb{N}_{\beta}} \in$ $A(N, M)$ and $n \geq T$

$$
\begin{aligned}
& \left|\frac{S_{L} x_{n}}{n^{2}}-\frac{S_{L} y_{n}}{n^{2}}\right| \\
& \leq \frac{1}{n^{2}} \sum_{i=1}^{\infty} \sum_{u=n+(2 i-1) \tau}^{n+2 i \tau-1} \sum_{s=u}^{\infty}\left[\mid h\left(s, x_{h_{1 s}}, x_{h_{2 s}}, \ldots, x_{h_{k s}}\right)\right. \\
& -h\left(s, y_{h_{1 s}}, y_{h_{2 s}}, \ldots, y_{h_{k s}}\right) \\
& +\sum_{t=s}^{\infty} \mid f\left(t, x_{f_{1 t}}, x_{f_{2 t}}, \ldots, x_{f_{k t}}\right) \\
& \left.-f\left(t, y_{f_{1 t}}, y_{f_{2 t}}, \ldots, y_{f_{k t}}\right) \mid\right] \\
& \leq \frac{\|x-y\|}{n^{2}} \sum_{i=1}^{\infty} \sum_{u=n+(2 i-1) \tau}^{n+2 i \tau-1} \sum_{s=u}^{\infty}\left(R_{s} H_{s}+\sum_{t=s}^{\infty} P_{t} F_{t}\right) \\
& \leq \frac{\|x-y\|}{T^{2}} \sum_{u=T}^{\infty} \sum_{s=u}^{\infty}\left(R_{s} H_{s}+\sum_{t=s}^{\infty} P_{t} F_{t}\right)=\theta\|x-y\|,
\end{aligned}
$$

$$
\begin{aligned}
& \left|\frac{S_{L} x_{n}}{n^{2}}-L\right| \\
& \leq \frac{1}{n^{2}} \sum_{i=1}^{\infty} \sum_{u=n+(2 i-1) \tau}^{n+2 i \tau-1} \sum_{s=u}^{\infty}\left\{\left|h\left(s, x_{h_{1 s}}, x_{h_{2 s}}, \ldots, x_{h_{k s}}\right)\right|\right. \\
& +\sum_{t=s}^{\infty}\left[\left|f\left(t, x_{f_{1 t}}, x_{f_{2 t}}, \ldots, x_{f_{k t}}\right)\right|\right. \\
& \left.\left.+\left|c_{t}\right|\right]\right\} \\
& \leq \frac{1}{T^{2}} \sum_{u=T}^{\infty} \sum_{s=u}^{\infty}\left(W_{s}+\sum_{t=s}^{\infty}\left[W_{s}+\sum_{t=s}^{\infty}\left(Q_{t}+\left|c_{t}\right|\right)\right]\right) \\
& <\min \{M-L, L-N\},
\end{aligned}
$$

which imply (27). The rest of the proof is similar to the proof of Theorem 2 and is omitted. This completes the proof.

Theorem 4. Assume that there exist three constants $b, M$, and $N$ with $(1-b) M>N>0$ and four nonnegative sequences $\left\{P_{n}\right\}_{n \in \mathbb{N}_{n_{0}}},\left\{Q_{n}\right\}_{n \in \mathbb{N}_{n_{0}}},\left\{R_{n}\right\}_{n \in \mathbb{N}_{n_{0}}}$ and $\left\{W_{n}\right\}_{n \in \mathbb{N}_{n_{0}}}$ satisfying (14), (15), (38), (39) and

$$
0 \leq b_{n} \leq b<1 \text { eventually. }
$$

Then one has the following.

(a) For any $L \in(b M+N, M)$, there exist $\theta \in$ $(0,1)$ and $T \geq n_{0}+\tau+\beta$ such that, for any $x_{0}=\left\{x_{0 n}\right\}_{n \in \mathbb{N}_{\beta}} \in A(N, M)$, the Mann iterative sequence $\left\{x_{m}\right\}_{m \in \mathbb{N}_{0}}=\left\{\left\{x_{m n}\right\}_{n \in \mathbb{N}_{\beta}}\right\}_{m \in \mathbb{N}_{0}}$ generated by the scheme

$x_{m+1 n}$

$$
\left\{\begin{array}{c}
\left(1-\alpha_{m}\right) x_{m n} \\
+\alpha_{m}\left\{n^{2} L-b_{n} x_{m n-\tau}\right. \\
-\sum_{u=n}^{\infty} \sum_{s=u}^{\infty}\left[h\left(s, x_{m h_{1 s}}, x_{m h_{2 s}}, \ldots, x_{m h_{k s}}\right)\right. \\
-\sum_{t=s}^{\infty}\left(f\left(t, x_{m f_{1 t}}, x_{m f_{2 t}}, \ldots, x_{m f_{k t}}\right)\right. \\
\left.\left.\left.-c_{t}\right)\right]\right\}, \\
n \geq T, \quad m \geq 0, \\
\left(1-\alpha_{m}\right) x_{m T} \\
+\alpha_{m}\left\{T^{2} L-b_{T} x_{m T-\tau}, x^{\infty} \sum_{u=T}^{\infty}\left[h\left(s, x_{m h_{1 s}}, x_{m h_{2 s}}, \ldots, x_{m h_{k s}}\right)\right.\right. \\
-\sum_{t=s}^{\infty}\left(f\left(t, x_{m f_{1 t}}, x_{m f_{2 t}}, \ldots, x_{m f_{k t}}\right)\right. \\
\left.\left.\left.\quad-c_{t}\right)\right]\right\}, \\
\beta \leq n<T, \quad m \geq 0,
\end{array}\right.
$$

converges to a positive solution $z=\left\{z_{n}\right\}_{n \in \mathbb{N}_{\beta}} \in$ $A(N, M)$ of (6) with $\lim _{n \rightarrow \infty} z_{n}=+\infty$ and has the 
error estimate (20), where $\left\{\alpha_{m}\right\}_{m \in \mathbb{N}_{0}}$ is an arbitrary sequence in $[0,1]$ satisfying $(21)$.

(b) Equation (6) possesses uncountably many positive solutions in $A(N, M)$.

Proof. Put $L \in(b M+N, M)$. It follows from (38), (39), and (47) that there exist $\theta \in(0,1)$ and $T \geq n_{0}+\tau+\beta$ satisfying

$$
\begin{gathered}
\theta=b+\frac{1}{T^{2}} \sum_{u=T}^{\infty} \sum_{s=u}^{\infty}\left(R_{s} H_{s}+\sum_{t=s}^{\infty} P_{t} F_{t}\right) ; \\
\frac{1}{T^{2}} \sum_{u=T}^{\infty} \sum_{s=u}^{\infty}\left(W_{s}+\sum_{t=s}^{\infty}\left(Q_{t}+\left|c_{t}\right|\right)\right) \\
<\min \{M-L, L-b M-N\} ; \\
0 \leq b_{n} \leq b<1, \quad \forall n \geq T .
\end{gathered}
$$

Define a mapping $S_{L}: A(N, M) \rightarrow l_{\beta}^{\infty}$ by

$$
\begin{aligned}
& S_{L} x_{n} \\
& =\left\{\begin{array}{r}
n^{2} L-b_{n} x_{n-\tau} \\
-\sum_{u=n}^{\infty} \sum_{s=u}^{\infty}\left\{h\left(s, x_{h_{1 s}}, x_{h_{2 s}}, \ldots, x_{h_{k s}}\right)\right. \\
\left.-\sum_{t=s}^{\infty}\left[f\left(t, x_{f_{1 t}}, x_{f_{2 t}}, \ldots, x_{f_{k t}}\right)-c_{t}\right]\right\}, \\
n \geq T, \\
S_{L} x_{T}, \quad \beta \leq n<T,
\end{array}\right.
\end{aligned}
$$

for each $x=\left\{x_{n}\right\}_{n \in \mathbb{N}_{\beta}} \in A(N, M)$. In view of (14), (15), and (49) and (50), we obtain that for each $x=\left\{x_{n}\right\}_{n \in \mathbb{N}_{\beta}}, y=$ $\left\{y_{n}\right\}_{n \in \mathbb{N}_{\beta}} \in A(N, M)$ and $n \geq T$

$$
\begin{aligned}
& \left|\frac{S_{L} x_{n}}{n^{2}}-\frac{S_{L} y_{n}}{n^{2}}\right| \\
& \leq b_{n}\left|\frac{x_{n-\tau}-y_{n-\tau}}{n^{2}}\right| \\
& +\frac{1}{n^{2}} \sum_{u=n}^{\infty} \sum_{s=u}^{\infty}\left[\mid h\left(s, x_{h_{1 s}}, x_{h_{2 s}}, \ldots, x_{h_{k s}}\right)\right. \\
& -h\left(s, y_{h_{1 s}}, y_{h_{2 s}}, \ldots, y_{h_{k s}}\right) \mid \\
& +\sum_{t=s}^{\infty} \mid f\left(t, x_{f_{1 t}}, x_{f_{2 t}}, \ldots, x_{f_{k t}}\right) \\
& \left.-f\left(t, y_{f_{1 t}}, y_{f_{2 t}}, \ldots, y_{f_{k t}}\right) \mid\right] \\
& \leq b_{n}\left|\frac{x_{n-\tau}-y_{n-\tau}}{(n-\tau)^{2}}\right| \frac{(n-\tau)^{2}}{n^{2}} \\
& +\frac{1}{n^{2}} \sum_{u=n}^{\infty} \sum_{s=u}^{\infty}\left[R_{s} \max \left\{\left|x_{h_{l s}}-y_{h_{l s}}\right|: 1 \leq l \leq k\right\}\right. \\
& \left.+\sum_{t=s}^{\infty} P_{t} \max \left\{\left|x_{f_{l t}}-y_{f_{l t}}\right|: 1 \leq l \leq k\right\}\right]
\end{aligned}
$$

$$
\begin{aligned}
& \leq b\|x-y\| \\
& +\frac{\|x-y\|}{n^{2}} \sum_{u=n}^{\infty} \sum_{s=u}^{\infty}\left[R_{s} \max \left\{h_{l s}^{2}: 1 \leq l \leq k\right\}\right. \\
& \left.+\sum_{t=s}^{\infty} P_{t} \max \left\{f_{l t}^{2}: 1 \leq l \leq k\right\}\right] \\
& \leq\left[b+\frac{1}{T^{2}} \sum_{u=T}^{\infty} \sum_{s=u}^{\infty}\left(R_{s} H_{s}+\sum_{t=s}^{\infty} P_{t} F_{t}\right)\right]\|x-y\|=\theta\|x-y\|, \\
& \frac{S_{L} x_{n}}{n^{2}} \\
& \leq L+\frac{1}{n^{2}} \sum_{u=n}^{\infty} \sum_{s=u}^{\infty}\left\{\left|h\left(s, x_{h_{1 s}}, x_{h_{2 s}}, \ldots, x_{h_{k s}}\right)\right|\right. \\
& \left.+\sum_{t=s}^{\infty}\left[\left|f\left(t, x_{f_{1 t}}, x_{f_{2 t}}, \ldots, x_{f_{k t}}\right)\right|+\left|c_{t}\right|\right]\right\} \\
& \leq L+\frac{1}{T^{2}} \sum_{u=T}^{\infty} \sum_{s=u}^{\infty}\left(W_{s}+\sum_{t=s}^{\infty}\left(Q_{t}+\left|c_{t}\right|\right)\right) \\
& <L+\min \{M-L, L-b M-N\} \leq M, \\
& \frac{S_{L} x_{n}}{n^{2}} \\
& \geq L-b M \\
& -\frac{1}{n^{2}} \sum_{u=n}^{\infty} \sum_{s=u}^{\infty}\left\{\left|h\left(s, x_{h_{1 s}}, x_{h_{2 s}}, \ldots, x_{h_{k s}}\right)\right|\right. \\
& \left.+\sum_{t=s}^{\infty}\left[\left|f\left(t, x_{f_{1 t}}, x_{f_{2 t}}, \ldots, x_{f_{k t}}\right)\right|+\left|c_{t}\right|\right]\right\} \\
& \geq L-b M-\frac{1}{T^{2}} \sum_{u=T}^{\infty} \sum_{s=u}^{\infty}\left[W_{s}+\sum_{t=s}^{\infty}\left(Q_{t}+\left|c_{t}\right|\right)\right] \\
& >L-b M-\min \{M-L, L-b M-N\} \geq N \text {, }
\end{aligned}
$$

which imply (27). The rest of the proof is similar to that of Theorem 2 and is omitted. This completes the proof.

Theorem 5. Assume that there exist constants $b, M$, and $N$ with $(1+b) M>N>0$ and four nonnegative sequences $\left\{P_{n}\right\}_{n \in \mathbb{N}_{n_{0}}},\left\{Q_{n}\right\}_{n \in \mathbb{N}_{n_{0}}},\left\{R_{n}\right\}_{n \in \mathbb{N}_{n_{0}}}$, and $\left\{W_{n}\right\}_{n \in \mathbb{N}_{n_{0}}}$ satisfying (14), (15), (38), (39), and

$$
-1<b \leq b_{n} \leq 0 \quad \text { eventually. }
$$

Then one has the following.

(a) For any $L \in(N,(1+b) M)$, there exist $\theta \in$ $(0,1)$ and $T \geq n_{0}+\tau+\beta$ such that, for any $x_{0}=\left\{x_{0 n}\right\}_{n \in \mathbb{N}_{\beta}} \in A(N, M)$, the Mann iterative sequence $\left\{x_{m}\right\}_{m \in \mathbb{N}_{0}}=\left\{\left\{x_{m n}\right\}_{n \in \mathbb{N}_{\beta}}\right\}_{m \in \mathbb{N}_{0}}$ generated by (48) converges to a positive solution $z=\left\{z_{n}\right\}_{n \in \mathbb{N}_{\beta}} \in$ 
$A(N, M)$ of (6) with $\lim _{n \rightarrow \infty} z_{n}=+\infty$ and has the error estimate (20), where $\left\{\alpha_{m}\right\}_{m \in \mathbb{N}_{0}}$ is an arbitrary sequence in $[0,1]$ satisfying $(21)$.

(b) Equation (6) possesses uncountably many positive solutions in $A(N, M)$.

Proof. Put $L \in(N,(1+b) M)$. It follows from (38), (39), and (52) that there exist $\theta \in(0,1)$ and $T \geq n_{0}+\tau+\beta$ satisfying

$$
\begin{gathered}
\theta=-b+\frac{1}{T^{2}} \sum_{u=T}^{\infty} \sum_{s=u}^{\infty}\left(R_{s} H_{s}+\sum_{t=s}^{\infty} P_{t} F_{t}\right) \\
\frac{1}{T^{2}} \sum_{u=T}^{\infty} \sum_{s=u}^{\infty}\left(W_{s}+\sum_{t=s}^{\infty}\left(Q_{t}+\left|c_{t}\right|\right)\right) \\
<\min \{(1+b) M-L, L-N\} \\
-1<b \leq b_{n} \leq 0, \quad \forall n \geq T .
\end{gathered}
$$

Define a mapping $S_{L}: A(N, M) \rightarrow l_{\beta}^{\infty}$ by (50). By virtue of (15), (50), (53), and (55), we infer that for all $x=\left\{x_{n}\right\}_{n \in \mathbb{N}_{\beta}}$, $y=\left\{y_{n}\right\}_{n \in \mathbb{N}_{\beta}} \in A(N, M)$ and $n \geq T$

$$
\begin{aligned}
& \left|\frac{S_{L} x_{n}}{n^{2}}-\frac{S_{L} y_{n}}{n^{2}}\right| \\
& \leq b_{n}\left|\frac{x_{n-\tau}-y_{n-\tau}}{n^{2}}\right| \\
& +\frac{1}{n^{2}} \sum_{u=n}^{\infty} \sum_{s=u}^{\infty}\left[\mid h\left(s, x_{h_{1 s}}, x_{h_{2 s}}, \ldots, x_{h_{k s}}\right)\right. \\
& -h\left(s, y_{h_{1 s}}, y_{h_{2 s}}, \ldots, y_{h_{k s}}\right) \mid \\
& +\sum_{t=s}^{\infty} \mid f\left(t, x_{f_{1 t}}, x_{f_{2 t}}, \ldots, x_{f_{k t}}\right) \\
& \left.-f\left(t, y_{f_{1 t}}, y_{f_{2 t}}, \ldots, y_{f_{k t}}\right) \mid\right] \\
& \leq\left[-b+\frac{1}{T^{2}} \sum_{u=T}^{\infty} \sum_{s=u}^{\infty}\left(R_{s} H_{s}+\sum_{t=s}^{\infty} P_{t} F_{t}\right)\right]\|x-y\| \\
& =\theta\|x-y\| \text {, } \\
& \frac{S_{L} x_{n}}{n^{2}} \\
& \leq L-b M \\
& +\frac{1}{n^{2}} \sum_{u=n}^{\infty} \sum_{s=u}^{\infty}\left\{\left|h\left(s, x_{h_{1 s}}, x_{h_{2 s}}, \ldots, x_{h_{k s}}\right)\right|\right. \\
& \left.+\sum_{t=s}^{\infty}\left[\left|f\left(t, x_{f_{1 t}}, x_{f_{2 t}}, \ldots, x_{f_{k t}}\right)\right|+\left|c_{t}\right|\right]\right\}
\end{aligned}
$$

$$
\begin{aligned}
& \leq L-b M+\frac{1}{T^{2}} \sum_{u=T}^{\infty} \sum_{s=u}^{\infty}\left(W_{s}+\sum_{t=s}^{\infty}\left(Q_{t}+\left|c_{t}\right|\right)\right) \\
& <L-b M+\min \{(1+b) M-L, L-N\} \leq M \\
& \frac{S_{L} x_{n}}{n^{2}} \\
& \geq L-\frac{1}{n^{2}} \sum_{u=n}^{\infty} \sum_{s=u}^{\infty}\left\{\left|h\left(s, x_{h_{1 s}}, x_{h_{2 s}}, \ldots, x_{h_{k s}}\right)\right|\right. \\
& \left.\quad+\sum_{t=s}^{\infty}\left[\left|f\left(t, x_{f_{1 t}}, x_{f_{2 t}}, \ldots, x_{f_{k t}}\right)\right|+\left|c_{t}\right|\right]\right\} \\
& \geq L-\frac{1}{T^{2}} \sum_{u=T}^{\infty} \sum_{s=u}^{\infty}\left[W_{s}+\sum_{t=s}^{\infty}\left(Q_{t}+\left|c_{t}\right|\right)\right] \\
& >L-\min \{(1+b) M-L, L-N\} \geq N .
\end{aligned}
$$

That is, (27) holds. The rest of the proof is similar to that of Theorem 2 and is omitted. This completes the proof.

Theorem 6. Assume that there exist constants $b$, $M$, and $N$ with $(1-1 / b) M>N>0$ and four nonnegative sequences $\left\{P_{n}\right\}_{n \in \mathbb{N}_{n_{0}}}, \quad\left\{Q_{n}\right\}_{n \in \mathbb{N}_{n_{0}}}, \quad\left\{R_{n}\right\}_{n \in \mathbb{N}_{n_{0}}}$, and $\left\{W_{n}\right\}_{n \in \mathbb{N}_{n_{0}}}$ satisfying (14), (15), (38), (39), and

$$
b_{n} \geq b>1 \text { eventually. }
$$

Then one has the following.

(a) For any $L \in((1 / b) M+N, M)$, there exist $\theta \in$ $(0,1)$ and $T \geq n_{0}+\tau+\beta$ such that, for any $x_{0}=$ $\left\{x_{0 n}\right\}_{n \in \mathbb{N}_{\beta}} \in A(N, M)$, the Mann iterative sequence $\left\{x_{m}\right\}_{m \in \mathbb{N}_{0}}=\left\{\left\{x_{m n}\right\}_{n \in \mathbb{N}_{\beta}}\right\}_{m \in \mathbb{N}_{0}}$ generated by the scheme

$x_{m+1 n}$

$$
\begin{aligned}
& \left\{\begin{array}{l}
\left(1-\alpha_{m}\right) x_{m n} \\
+\alpha_{m}\left\{n^{2} L-\frac{x_{m n+\tau}}{b_{n+\tau}}-\frac{1}{b_{n+\tau}}\right.
\end{array}\right. \\
& \times \sum_{u=n+\tau}^{\infty} \sum_{s=u}^{\infty}\left[h\left(s, x_{m h_{1 s}}, x_{m h_{2 s}}, \ldots, x_{m h_{k s}}\right)\right. \\
& -\sum_{t=s}^{\infty}\left(f\left(t, x_{m f_{1 t}}, x_{m f_{2 t}}, \ldots, x_{m f_{k t}}\right)\right. \\
& \left.\left.\left.-c_{t}\right)\right]\right\} \text {, } \\
& =\left\{\begin{array}{l}
\left(1-\alpha_{m}\right) x_{m T} \\
+\alpha_{m}\left\{T^{2} L-\frac{x_{m T+\tau}}{b_{T+\tau}}\right.
\end{array}\right. \\
& -\sum_{u=T+\tau}^{\infty} \sum_{s=u}^{\infty}\left[h\left(s, x_{m h_{1 s}}, x_{m h_{2 s}}, \ldots, x_{m h_{k s}}\right)\right. \\
& -\sum_{t=s}^{\infty}\left(f\left(t, x_{m f_{1 t}}, x_{m f_{2 t}}, \ldots, x_{m f_{k t}}\right)\right. \\
& \left.\left.\left.-c_{t}\right)\right]\right\} \text {, } \\
& \beta \leq n<T, \quad m \geq 0 \text {, }
\end{aligned}
$$


converges to a positive solution $z=\left\{z_{n}\right\}_{n \in \mathbb{N}_{\beta}} \epsilon$ $A(N, M)$ of (6) with $\lim _{n \rightarrow \infty} z_{n}=+\infty$ and has the error estimate (20), where $\left\{\alpha_{m}\right\}_{m \in \mathbb{N}_{0}}$ is an arbitrary sequence in $[0,1]$ satisfying $(21)$.

(b) Equation (6) possesses uncountably many positive solutions in $A(N, M)$.

Proof. Put $L \in((1 / b) M+N, M)$. It follows from (38), (39), and (57) that there exist $\theta \in(0,1)$ and $T \geq n_{0}+\tau+$ $\beta$ satisfying

$$
\begin{gathered}
\theta=\frac{1}{b}\left[\left(1+\frac{\tau}{T}\right)^{2}+\frac{1}{T^{2}} \sum_{u=T}^{\infty} \sum_{s=u}^{\infty}\left(R_{s} H_{s}+\sum_{t=s}^{\infty} P_{t} F_{t}\right)\right] ; \\
\frac{1}{b T^{2}} \sum_{u=T}^{\infty} \sum_{s=u}^{\infty}\left(W_{s}+\sum_{t=s}^{\infty}\left(Q_{t}+\left|c_{t}\right|\right)\right) \\
<\min \left\{M-L, L-\frac{1}{b} M-N\right\} ; \\
b_{n} \geq b>1, \quad \forall n \geq T .
\end{gathered}
$$

Define a mapping $S_{L}: A(N, M) \rightarrow l_{\beta}^{\infty}$ by

$$
\begin{aligned}
& S_{L} x_{n} \\
& =\left\{\begin{array}{r}
n^{2} L-\frac{x_{n+\tau}}{b_{n+\tau}}-\frac{1}{b_{n+\tau}} \\
\times \sum_{u=n+\tau}^{\infty} \sum_{s=u}^{\infty}\left\{h\left(s, x_{h_{1 s}}, x_{h_{2 s}}, \ldots, x_{h_{k s}}\right)\right. \\
-\sum_{t=s}^{\infty}\left[\left(f\left(t, x_{f_{l t}}, x_{f_{2 t}}, \ldots, x_{f_{k t}}\right)\right.\right. \\
\left.\left.\left.-c_{t}\right)\right]\right\}, \quad n \geq T, \\
\beta \leq n<T,
\end{array}\right. \\
& S_{L} x_{T}, \quad n \geq T
\end{aligned}
$$

for each $x=\left\{x_{n}\right\}_{n \in \mathbb{N}_{\beta}} \in A(N, M)$. In view of (14), (15), and (59) $\backsim(62)$, we obtain that for each $x=\left\{x_{n}\right\}_{n \in \mathbb{N}_{\beta}}, y=$ $\left\{y_{n}\right\}_{n \in \mathbb{N}_{\beta}} \in A(N, M)$ and $n \geq T$

$$
\begin{aligned}
&\left|\frac{S_{L} x_{n}}{n^{2}}-\frac{S_{L} y_{n}}{n^{2}}\right| \\
& \leq \frac{1}{b_{n+\tau}}\left|\frac{x_{n+\tau}-y_{n+\tau}}{n^{2}}\right| \\
&+\frac{1}{b_{n+\tau} n^{2}} \sum_{u=n+\tau}^{\infty} \sum_{s=u}^{\infty}\left[\mid h\left(s, x_{h_{1 s}}, x_{h_{2 s}}, \ldots, x_{h_{k s}}\right)\right. \\
&-h\left(s, y_{h_{1 s}}, y_{h_{2 s}}, \ldots, y_{h_{k s}}\right) \mid \\
&+\sum_{t=s}^{\infty} \mid f\left(t, x_{f_{1 t}}, x_{f_{2 t}}, \ldots, x_{f_{k t}}\right) \\
&\left.\quad-f\left(t, y_{f_{1 t}}, y_{f_{2 t}}, \ldots, y_{f_{k t}}\right) \mid\right]
\end{aligned}
$$

$$
\begin{aligned}
& \leq \frac{1}{b_{n+\tau}}\left|\frac{x_{n+\tau}-y_{n+\tau}}{(n+\tau)^{2}}\right| \frac{(n+\tau)^{2}}{n^{2}} \\
& +\frac{1}{b_{n+\tau} n^{2}} \sum_{u=n}^{\infty} \sum_{s=u}^{\infty}\left[R_{s} \max \left\{\left|x_{h_{l s}}-y_{h_{l s}}\right|: 1 \leq l \leq k\right\}\right. \\
& \left.+\sum_{t=s}^{\infty} P_{t} \max \left\{\left|x_{f_{t t}}-y_{f_{t t}}\right|: 1 \leq l \leq k\right\}\right] \\
& \leq \frac{1}{b}\left[\left(1+\frac{\tau}{T}\right)^{2}+\frac{1}{T^{2}} \sum_{u=T}^{\infty} \sum_{s=u}^{\infty}\left(R_{s} H_{s}+\sum_{t=s}^{\infty} P_{t} F_{t}\right)\right]\|x-y\| \\
& =\theta\|x-y\| \text {, } \\
& \frac{S_{L} x_{n}}{n^{2}} \\
& \leq L+\frac{1}{b n^{2}} \sum_{u=n+\tau}^{\infty} \sum_{s=u}^{\infty}\left\{\left|h\left(s, x_{h_{1 s}}, x_{h_{2 s}}, \ldots, x_{h_{k s}}\right)\right|\right. \\
& +\sum_{t=s}^{\infty}\left[\left|f\left(t, x_{f_{1 t}}, x_{f_{2 t}}, \ldots, x_{f_{k t}}\right)\right|\right. \\
& \left.\left.+\left|c_{t}\right|\right]\right\} \\
& \leq L+\frac{1}{b T^{2}} \sum_{u=T}^{\infty} \sum_{s=u}^{\infty}\left(W_{s}+\sum_{t=s}^{\infty}\left(Q_{t}+\left|c_{t}\right|\right)\right) \\
& <L+\min \left\{M-L, L-\frac{1}{b} M-N\right\} \leq M, \\
& \frac{S_{L} x_{n}}{n^{2}} \\
& \geq L-\frac{1}{b} M \\
& -\frac{1}{b n^{2}} \sum_{u=n+\tau}^{\infty} \sum_{s=u}^{\infty}\left\{\left|h\left(s, x_{h_{1 s}}, x_{h_{2 s}}, \ldots, x_{h_{k s}}\right)\right|\right. \\
& \left.+\sum_{t=s}^{\infty}\left[\left|f\left(t, x_{f_{1 t}}, x_{f_{2 t}}, \ldots, x_{f_{k t}}\right)\right|+\left|c_{t}\right|\right]\right\} \\
& \geq L-\frac{1}{b} M-\frac{1}{b T^{2}} \sum_{u=T}^{\infty} \sum_{s=\mathcal{u}}^{\infty}\left(W_{s}+\sum_{t=s}^{\infty}\left(Q_{t}+\left|c_{t}\right|\right)\right) \\
& >L-\frac{1}{b} M-\min \left\{M-L, L-\frac{1}{b} M-N\right\} \geq N,
\end{aligned}
$$

which imply (27). The rest of the proof is similar to that of Theorem 2 and is omitted. This completes the proof.

Theorem 7. Assume that there exist constants $b, M$, and $N$ with $(1+1 / b) M>N>0$ and four nonnegative sequences $\left\{P_{n}\right\}_{n \in \mathbb{N}_{n_{0}}}, \quad\left\{Q_{n}\right\}_{n \in \mathbb{N}_{n_{0}}}, \quad\left\{R_{n}\right\}_{n \in \mathbb{N}_{n_{0}}}$, and $\left\{W_{n}\right\}_{n \in \mathbb{N}_{n_{0}}}$ satisfying (14), (15), (38), (39) and

$$
b_{n} \leq b<-1 \text { eventually. }
$$


Then one has the following.

(a) For any $L \in(-(1+1 / b) M,-N)$, there exist $\theta \in(0,1)$ and $T \geq n_{0}+\tau+\beta$ such that, for any $x_{0}=\left\{x_{0 n}\right\}_{n \in \mathbb{N}_{\beta}} \in$ $A(N, M)$, the Mann iterative sequence $\left\{x_{m}\right\}_{m \in \mathbb{N}_{0}}=$ $\left\{\left\{x_{m n}\right\}_{n \in \mathbb{N}_{\beta}}\right\}_{m \in \mathbb{N}_{0}}$ generated by the scheme

$x_{m+1 n}$

$$
\begin{aligned}
& \left\{\begin{aligned}
&\left(1-\alpha_{m}\right) x_{m n} \\
&+\alpha_{m}\{-n^{2} L-\frac{x_{m n+\tau}}{b_{n+\tau}}-\frac{1}{b_{n+\tau}} \\
& \times \sum_{u=n+\tau}^{\infty} \sum_{s=u}^{\infty}\left[h\left(s, x_{m h_{1 s}}, x_{m h_{2 s}}, \ldots, x_{m h_{k s}}\right)\right.
\end{aligned}\right. \\
& -\sum_{t=s}^{\infty}\left(f\left(t, x_{m f_{1 t}}, x_{m f_{2 t}}, \ldots, x_{m f_{k t}}\right)\right. \\
& \left.\left.\left.-c_{t}\right)\right]\right\} \text {, } \\
& n \geq T, \quad m \geq 0 \text {, } \\
& =\left\{\begin{array}{l}
\left(1-\alpha_{m}\right) x_{m T} \\
+\alpha_{m}\left\{-T^{2} L-\frac{x_{m T+\tau}}{b_{T+\tau}}\right.
\end{array}\right. \\
& -\sum_{u=T+\tau}^{\infty} \sum_{s=u}^{\infty}\left[h\left(s, x_{m h_{1 s}}, x_{m h_{2 s}}, \ldots, x_{m h_{k s}}\right)\right. \\
& -\sum_{t=s}^{\infty}\left(f\left(t, x_{m f_{1 t}}, x_{m f_{2 t}}, \ldots, x_{m f_{k t}}\right)\right. \\
& \left.\left.\left.-c_{t}\right)\right]\right\}, \\
& \beta \leq n<T, \quad m \geq 0 \text {, }
\end{aligned}
$$

converges to a positive solution $z=\left\{z_{n}\right\}_{n \in \mathbb{N}_{\beta}} \in$ $A(N, M)$ of (6) with $\lim _{n \rightarrow \infty} z_{n}=+\infty$ and has the error estimate (20), where $\left\{\alpha_{m}\right\}_{m \in \mathbb{N}_{0}}$ is an arbitrary sequence in $[0,1]$ satisfying $(21)$.

(b) Equation (6) possesses uncountably many positive solutions in $A(N, M)$.

Proof. Put $L \in(-(1+1 / b) M,-N)$. It follows from (38), (39), and (64) that there exist $\theta \in(0,1)$ and $T \geq n_{0}+\tau+$ $\beta$ satisfying

$$
\begin{gathered}
\theta=-\frac{1}{b}\left[\left(1+\frac{\tau}{T}\right)^{2}+\frac{1}{T^{2}} \sum_{u=T}^{\infty} \sum_{s=u}^{\infty}\left(R_{s} H_{s}+\sum_{t=s}^{\infty} P_{t} F_{t}\right)\right] \\
-\frac{1}{b T^{2}} \sum_{u=T}^{\infty} \sum_{s=u}^{\infty}\left(W_{s}+\sum_{t=s}^{\infty}\left(Q_{t}+\left|c_{t}\right|\right)\right) \\
<\min \left\{\left(1+\frac{1}{b}\right) M-L, L-\frac{1}{b} M-N\right\} \\
b_{n} \geq b>1, \quad \forall n \geq T .
\end{gathered}
$$

Define a mapping $S_{L}: A(N, M) \rightarrow l_{\beta}^{\infty}$ by

$$
\begin{aligned}
& S_{L} x_{n} \\
& =\left\{\begin{array}{rr}
-n^{2} L-\frac{x_{n+\tau}}{b_{n+\tau}}-\frac{1}{b_{n+\tau}} & \\
\times \sum_{u=n+\tau}^{\infty} \sum_{s=u}^{\infty}\left\{h\left(s, x_{h_{1 s}}, x_{h_{2 s}}, \ldots, x_{h_{k s}}\right)\right. & n \geq T, \\
\left.-\sum_{t=s}^{\infty}\left[f\left(t, x_{f_{1 t}}, x_{f_{2 t}}, \ldots, x_{f_{k t}}\right)-c_{t}\right]\right\}, & \\
S_{L} x_{T}, & \beta \leq n<T,
\end{array}\right.
\end{aligned}
$$

for each $x=\left\{x_{n}\right\}_{n \in \mathbb{N}_{\beta}} \in A(N, M)$. Making use of (15), (66), (68), and (69), we conclude that

$$
\begin{aligned}
& \left|\frac{S_{L} x_{n}}{n^{2}}-\frac{S_{L} y_{n}}{n^{2}}\right| \\
& \leq-\frac{1}{b_{n+\tau}}\left|\frac{x_{n+\tau}-y_{n+\tau}}{n^{2}}\right| \\
& -\frac{1}{b_{n+\tau} n^{2}} \sum_{u=n+\tau}^{\infty} \sum_{s=u}^{\infty}\left[\mid h\left(s, x_{h_{1 s}}, x_{h_{2 s}}, \ldots, x_{h_{k s}}\right)\right. \\
& -h\left(s, y_{h_{1 s}}, y_{h_{2 s}}, \ldots, y_{h_{k s}}\right) \mid \\
& +\sum_{t=s}^{\infty} \mid f\left(t, x_{f_{1 t}}, x_{f_{2 t}}, \ldots, x_{f_{k t}}\right) \\
& \left.-f\left(t, y_{f_{1 t}}, y_{f_{2 t}}, \ldots, y_{f_{k t}}\right) \mid\right]
\end{aligned}
$$$$
\leq-\frac{1}{b_{n+\tau}}\left|\frac{x_{n+\tau}-y_{n+\tau}}{(n+\tau)^{2}}\right| \frac{(n+\tau)^{2}}{n^{2}}
$$$$
-\frac{1}{b_{n+\tau} n^{2}} \sum_{u=n}^{\infty} \sum_{s=\mathcal{u}}^{\infty}\left[R_{s} \max \left\{\left|x_{h_{l s}}-y_{h_{l s}}\right|: 1 \leq l \leq k\right\}\right.
$$$$
\left.+\sum_{t=s}^{\infty} P_{t} \max \left\{\left|x_{f_{l t}}-y_{f_{l t}}\right|: 1 \leq l \leq k\right\}\right]
$$$$
\leq-\frac{1}{b}\left[\left(1+\frac{\tau}{T}\right)^{2}+\frac{1}{T^{2}} \sum_{u=T}^{\infty} \sum_{s=u}^{\infty}\left(R_{s} H_{s}+\sum_{t=s}^{\infty} P_{t} F_{t}\right)\right]\|x-y\|
$$$$
=\theta\|x-y\|,
$$$$
\frac{S_{L} x_{n}}{n^{2}}
$$

$$
\begin{aligned}
& \leq-L-\frac{M}{b}- \frac{1}{b n^{2}} \\
& \times \sum_{u=n+\tau}^{\infty} \sum_{s=u}^{\infty}\left\{\left|h\left(s, x_{h_{1 s}}, x_{h_{2 s}}, \ldots, x_{h_{k s}}\right)\right|\right. \\
&\left.+\sum_{t=s}^{\infty}\left[\left|f\left(t, x_{f_{1 t}}, x_{f_{2 t}}, \ldots, x_{f_{k t}}\right)\right|+\left|c_{t}\right|\right]\right\}
\end{aligned}
$$




$$
\begin{aligned}
& \leq-L-\frac{M}{b}-\frac{1}{b T^{2}} \sum_{u=T}^{\infty} \sum_{s=u}^{\infty}\left(W_{s}+\sum_{t=s}^{\infty}\left(Q_{t}+\left|c_{t}\right|\right)\right) \\
& <-L-\frac{M}{b}+\min \left\{\left(1+\frac{1}{b}\right) M+L,-L-N\right\} \leq M, \\
& \frac{S_{L} x_{n}}{n^{2}} \\
& \geq-L \\
& +\frac{1}{b n^{2}} \sum_{u=n+\tau}^{\infty} \sum_{s=u}^{\infty}\left\{\left|h\left(s, x_{h_{1 s}}, x_{h_{2 s}}, \ldots, x_{h_{k s}}\right)\right|\right. \\
& \geq-L+\frac{1}{b T^{2}} \sum_{u=T}^{\infty} \sum_{s=u}^{\infty}\left[W_{s}+\sum_{t=s}^{\infty}\left(Q_{t}+\left|c_{t}\right|\right)\right] \\
& >-L-\min \left\{\left(1+\frac{1}{b}\right) M+L,-L-N\right\} \geq N,
\end{aligned}
$$

which yield (27). The rest of the proof is similar to that of Theorem 2 and is omitted. This completes the proof.

\section{Examples}

In this section, we suggest six examples to explain the results presented in Section 2.

Example 1. Consider the third order nonlinear neutral delay difference equation

$$
\begin{aligned}
& \Delta^{3}\left(x_{n}-x_{n-\tau}\right)+\Delta\left(\frac{\sin ^{2} x_{n-3}}{n^{7}}\right)+\frac{1}{\left(n^{9}+2 n^{5}+1\right)\left(1+x_{n^{2}}^{2}\right)} \\
& =\frac{n^{2}-2 n}{n^{8}+n^{3}+1}, \quad \forall n \geq 4,
\end{aligned}
$$

where $\tau \in \mathbb{N}$ is fixed. Let $n_{0}=4, k=1$, and $\beta=\min \{4-\tau, 1\}$, and let $M$ and $N$ be two positive constants with $M>N$ and

$$
\begin{gathered}
b_{n}=-1, \quad c_{n}=\frac{n^{2}-2 n}{n^{8}+n^{3}+1}, \\
f(n, u)=\frac{1}{\left(n^{9}+2 n^{5}+1\right)\left(1+u^{2}\right)}, \\
h(n, u)=\frac{\sin ^{2} u}{n^{7}}, \quad f_{1 n}=n^{2}, \quad F_{n}=n^{4}, \\
h_{1 n}=n-3, \quad H_{n}=(n-3)^{2}, \quad P_{n}=\frac{2 M}{n^{9}}, \\
Q_{n}=\frac{1}{n^{9}}, \quad R_{n}=\frac{2}{n^{7}}, \quad W_{n}=\frac{1}{n^{7}}, \\
\quad \forall(n, u) \in \mathbb{N}_{n_{0}} \times \mathbb{R} .
\end{gathered}
$$

It is easy to see that (14), (15), and (18) are satisfied. Note that

$$
\begin{aligned}
& \frac{1}{n^{2}} \sum_{t=n+\tau}^{\infty} t^{2} \max \left\{R_{t} H_{t}, W_{t}\right\} \\
& \quad=\frac{1}{n^{2}} \sum_{t=n+\tau}^{\infty} t^{2} \max \left\{\frac{2(t-3)^{2}}{t^{7}}, \frac{1}{t^{7}}\right\} \\
& \quad=\sum_{t=n+\tau}^{\infty} \frac{2(t-3)^{2}+1}{t^{5}} \\
& \leq \frac{2}{n^{2}} \sum_{t=n+\tau}^{\infty} \frac{1}{t^{3}} \longrightarrow 0 \text { as } n \longrightarrow \infty \\
& \frac{1}{n^{2}} \sum_{t=n+\tau}^{\infty} t^{3} \max \left\{P_{t} F_{t}, Q_{t},\left|c_{t}\right|\right\} \\
& \quad=\frac{1}{n^{2}} \sum_{t=n+\tau}^{\infty} t^{3} \max \left\{\frac{2 M}{t^{5}}, \frac{1}{t^{9}}, \frac{\left|t^{2}-2 t\right|}{t^{8}+t^{3}+1}\right\} \\
& \leq \frac{\max \{1,2 M\}}{n^{2}} \sum_{t=n+\tau}^{\infty} \frac{1}{t^{2}} \longrightarrow 0 \text { as } n \longrightarrow \infty
\end{aligned}
$$

which together with Lemma 1 yield that (16) and (17) hold. It follows from Theorem 2 that (71) possesses uncountably many positive solutions in $A(N, M)$. On the other hand, for any $L \in(N, M)$, there exist $\theta \in(0,1)$ and $T \geq n_{0}+\tau+\beta$ such that, for each $x_{0}=\left\{x_{0 n}\right\}_{n \in \mathbb{N}_{\beta}} \in A(N, M)$, the Mann iterative sequence $\left\{x_{m}\right\}_{m \in \mathbb{N}_{0}}=\left\{\left\{x_{m n}\right\}_{n \in \mathbb{N}_{\beta}}\right\}_{m \in \mathbb{N}_{0}}$ generated by (19) converges to a positive solution $z=\left\{z_{n}\right\}_{n \in \mathbb{N}_{\beta}} \in A(N, M)$ of (71) with $\lim _{n \rightarrow \infty} z_{n}=+\infty$ and has the error estimate (20), where $\left\{\alpha_{m}\right\}_{m \in \mathbb{N}_{0}}$ is an arbitrary sequence in $[0,1]$ satisfying (21).

Example 2. Consider the third order nonlinear neutral delay difference equation

$$
\begin{aligned}
& \Delta^{3}\left(x_{n}+x_{n-\tau}\right)+\Delta\left(\frac{\sin ^{2} x_{3 n^{3}+1}}{n^{3}\left(n^{6}+2\right)\left(1+x_{2 n^{2}-3}^{4}\right)}\right) \\
& \quad+\frac{(-1)^{n} n^{3}\left(x_{n^{2}-n-1}+x_{(n+1)(n+2)}\right)}{\left(n^{13}+n^{5}+1\right)\left(1+x_{n^{2}-n-1}^{2}+x_{(n+1)(n+2)}^{2}\right)} \\
& =\frac{n^{2}-\ln n}{n^{6}+n^{5}+1}, \quad \forall n \geq 5,
\end{aligned}
$$

where $\tau \in \mathbb{N}$ is fixed. Let $n_{0}=5, k=2$, and $\beta=5-\tau$, and let $M$ and $N$ be two positive constants with $M>N$ and

$$
\begin{gathered}
b_{n}=1, \quad c_{n}=\frac{n^{2}-\ln n}{n^{6}+n^{5}+1}, \\
f(n, u, v)=\frac{(-1)^{n} n^{3}(u+v)}{\left(n^{13}+n^{5}+1\right)\left(1+u^{2}+v^{2}\right)},
\end{gathered}
$$




$$
\begin{gathered}
h(n, u, v)=\frac{\sin ^{2} v}{n^{3}\left(n^{6}+2\right)\left(1+u^{4}\right)}, \quad f_{1 n}=n^{2}-n-1, \\
f_{2 n}=(n+1)(n+2), \\
F_{n}=(n+1)^{2}(n+2)^{2}, \quad h_{1 n}=2 n^{2}-3, \\
h_{2 n}=3 n^{3}+1, \\
H_{n}=\left(3 n^{3}+1\right)^{2}, \quad P_{n}=Q_{n}=\frac{4}{n^{10}}, \quad R_{n}=W_{n}=\frac{10}{n^{9}}, \\
\forall(n, u, v) \in \mathbb{N}_{n_{0}} \times \mathbb{R}^{2} .
\end{gathered}
$$

It is clear that (14), (15), and (40) are fulfilled. Note that

$$
\begin{aligned}
& \frac{1}{n^{2}} \sum_{u=n}^{\infty} \sum_{s=u}^{\infty} \max \left\{R_{s} H_{s}, W_{s}\right\} \\
& \quad=\frac{1}{n^{2}} \sum_{u=n}^{\infty} \sum_{s=u}^{\infty} \max \left\{\frac{10\left(3 s^{3}+1\right)^{2}}{s^{9}}, \frac{10}{s^{9}}\right\} \\
& \leq \frac{160}{n^{2}} \sum_{u=n}^{\infty} \sum_{s=u}^{\infty} \frac{1}{s^{3}} \leq \frac{160}{n^{2}} \sum_{s=n}^{\infty} \frac{1}{s^{2}} \longrightarrow 0 \text { as } n \longrightarrow \infty,
\end{aligned}
$$

which means that

$$
\lim _{n \rightarrow \infty} \frac{1}{n^{2}} \sum_{u=n}^{\infty} \sum_{s=u}^{\infty} \max \left\{R_{s} H_{s}, W_{s}\right\}=0 .
$$

Observe that

$$
\begin{aligned}
& \frac{1}{n^{2}} \sum_{u=n}^{\infty} \sum_{s=u}^{\infty} \sum_{t=s}^{\infty} \max \left\{P_{t} F_{t}, Q_{t},\left|c_{t}\right|\right\} \\
& =\frac{1}{n^{2}} \sum_{u=n}^{\infty} \sum_{s=u}^{\infty} \sum_{t=s}^{\infty} \max \left\{\frac{4(t+1)^{2}(t+2)^{2}}{t^{10}}, \frac{4}{t^{10}}, \frac{t^{2}-\ln t}{t^{6}+t^{5}+1}\right\} \\
& \leq \frac{196}{n^{2}} \sum_{u=n}^{\infty} \sum_{s=u}^{\infty} \sum_{t=s}^{\infty} \frac{1}{t^{4}}=\frac{196}{n^{2}} \sum_{u=n}^{\infty} \sum_{t=u}^{\infty} \frac{t-u+1}{t^{4}} \\
& \leq \frac{196}{n^{2}} \sum_{u=n}^{\infty} \sum_{t=u}^{\infty} \frac{1}{t^{3}} \leq \frac{196}{n^{2}} \sum_{t=n}^{\infty} \frac{1}{t^{2}} \longrightarrow 0 \text { as } n \longrightarrow \infty,
\end{aligned}
$$

which yields that

$$
\lim _{n \rightarrow \infty} \frac{1}{n^{2}} \sum_{u=n}^{\infty} \sum_{s=u}^{\infty} \sum_{t=s}^{\infty} \max \left\{P_{t} F_{t}, Q_{t},\left|c_{t}\right|\right\}=0 .
$$

Thus Theorem 3 guarantees that (74) possesses uncountably positive solutions in $A(N, M)$. On the other hand, for any $L \in(N, M)$, there exist $\theta \in(0,1)$ and $T \geq \tau+$ $n_{0}+\beta$ such that the Mann iterative sequence $\left\{x_{m}\right\}_{m \in \mathbb{N}_{0}}=$ $\left\{\left\{x_{m n}\right\}_{n \in \mathbb{N}_{\beta}}\right\}_{m \in \mathbb{N}_{0}}$ generated by (41) converges to a positive solution $z=\left\{z_{n}\right\}_{n \in \mathbb{N}_{\beta}} \in A(N, M)$ of (74) with $\lim _{n \rightarrow \infty} z_{n}=$ $+\infty$ and has the error estimate (20), where $\left\{\alpha_{m}\right\}_{m \in \mathbb{N}_{0}}$ is an arbitrary sequence in $[0,1]$ satisfying $(21)$.
Example 3. Consider the third order nonlinear neutral delay difference equation

$$
\begin{aligned}
& \Delta^{3}\left(x_{n}+\frac{1+3 \ln n}{2+4 \ln n} x_{n-\tau}\right) \\
& +\Delta\left(\frac{(-1)^{n} \sin \left(e^{-n^{2}\left|x_{5 n^{2}-3}\right|}\right)}{n^{15}-\sqrt{n}+3}\right. \\
& \left.\quad+\frac{n^{2}+(-1)^{n(n+1) / 2}}{\left(n^{12}+6 n^{10}+7\right) e^{\left|x_{2 n^{3}+1}\right|}}\right) \\
& +\frac{(-1)^{n}}{n^{6}\left(1+x_{n-3}^{2}\right)}-\frac{1}{\left(n^{7}+2 n^{4}-1\right)\left(1+x_{n+4}^{2}\right)} \\
& =\frac{3(-1)^{n} n^{2}}{9 n^{10} \ln ^{3} n}, \quad \forall n \geq 7,
\end{aligned}
$$

where $\tau \in \mathbb{N}$ is fixed. Let $n_{0}=7, k=2, b=3 / 4$, and $\beta=\min \{7-\tau, 4\}$, and let $M$ and $N$ be two positive constants with $M>4 N$ and

$$
\begin{gathered}
b_{n}=\frac{1+3 \ln n}{2+4 \ln n}, \quad c_{n}=\frac{3(-1)^{n} n^{2}}{9 n^{10} \ln ^{3} n}, \\
f(n, u, v)=\frac{(-1)^{n}}{n^{6}\left(1+u^{2}\right)}-\frac{1}{\left(n^{7}+2 n^{4}-1\right)\left(1+v^{2}\right)}, \\
h(n, u, v)=\frac{(-1)^{n} \sin \left(e^{-n^{2}|u|}\right)}{n^{15}-\sqrt{n}+3}+\frac{n^{2}+(-1)^{n(n+1) / 2}}{\left(n^{12}+6 n^{10}+7\right) e^{|v|}} \\
f_{1 n}=n-3, \quad f_{2 n}=n+4 \\
F_{n}=(n+4)^{2}, \quad h_{1 n}=5 n^{2}-3, \\
h_{2 n}=2 n^{3}+1, \quad H_{n}=\left(2 n^{3}+1\right)^{2}, \\
P_{n}=Q_{n}=\frac{3}{n^{6}}, \quad R_{n}=W_{n}=\frac{2}{n^{10}}, \\
\forall(n, u, v) \in \mathbb{N}_{n_{0}} \times \mathbb{R}^{2} .
\end{gathered}
$$

It is not difficult to verify that (14), (15), and (47) are fulfilled. Note that

$$
\begin{aligned}
\frac{1}{n^{2}} \sum_{u=n}^{\infty} \sum_{s=u}^{\infty} \max \left\{R_{s} H_{s}, W_{s}\right\} & \\
= & \frac{1}{n^{2}} \sum_{u=n}^{\infty} \sum_{s=u}^{\infty} \max \left\{\frac{2\left(2 s^{3}+1\right)^{2}}{s^{10}}, \frac{2}{s^{10}}\right\} \\
& \leq \frac{18}{n^{2}} \sum_{u=n}^{\infty} \sum_{s=u}^{\infty} \frac{1}{s^{4}} \leq \frac{18}{n^{2}} \sum_{s=n}^{\infty} \frac{1}{s^{3}} \longrightarrow 0 \quad \text { as } n \longrightarrow \infty,
\end{aligned}
$$

which implies that

$$
\lim _{n \rightarrow \infty} \frac{1}{n^{2}} \sum_{u=n}^{\infty} \sum_{s=u}^{\infty} \max \left\{R_{s} H_{s}, W_{s}\right\}=0 .
$$


Observe that

$$
\begin{aligned}
& \frac{1}{n^{2}} \sum_{u=n}^{\infty} \sum_{s=u}^{\infty} \sum_{t=s}^{\infty} \max \left\{P_{t} F_{t}, Q_{t},\left|c_{t}\right|\right\} \\
& \quad=\frac{1}{n^{2}} \sum_{u=n}^{\infty} \sum_{s=u}^{\infty} \sum_{t=s}^{\infty} \max \left\{\frac{3(t+4)^{2}}{t^{6}}, \frac{3}{t^{6}},\left|\frac{3(-1)^{t} t^{2}}{9 t^{10} \ln ^{3} t}\right|\right\} \\
& \leq \frac{12}{n^{2}} \sum_{u=n}^{\infty} \sum_{s=u}^{\infty} \sum_{t=s}^{\infty} \frac{1}{t^{4}} \\
& \leq \frac{12}{n^{2}} \sum_{u=n}^{\infty} \sum_{t=u}^{\infty} \frac{1}{t^{3}} \leq \frac{12}{n^{2}} \sum_{t=n}^{\infty} \frac{1}{t^{2}} \longrightarrow 0 \quad \text { as } n \longrightarrow \infty
\end{aligned}
$$

which means that

$$
\lim _{n \rightarrow \infty} \frac{1}{n^{2}} \sum_{u=n}^{\infty} \sum_{s=u}^{\infty} \sum_{t=s}^{\infty} \max \left\{P_{t} F_{t}, Q_{t},\left|c_{t}\right|\right\}=0 .
$$

That is, (38) and (39) hold. Consequently Theorem 4 implies that (80) possesses uncountably many positive solutions in $A(N, M)$. On the other hand, for any $L \in((3 / 4) M+$ $N, M)$, there exist $\theta \in(0,1)$ and $T \geq n_{0}+\tau+$ $\beta$ such that the Mann iterative sequence $\left\{x_{m}\right\}_{m \in \mathbb{N}_{0}}=$ $\left\{\left\{x_{m n}\right\}_{n \in \mathbb{N}_{\beta}}\right\}_{m \in \mathbb{N}_{0}}$ generated by (41) converges to a positive solution $z=\left\{z_{n}\right\}_{n \in \mathbb{N}_{\beta}} \in A(N, M)$ of (80) with $\lim _{n \rightarrow \infty} z_{n}=$ $+\infty$ and has the error estimate (20), where $\left\{\alpha_{m}\right\}_{m \in \mathbb{N}_{0}}$ is an arbitrary sequence in $[0,1]$ satisfying $(21)$.

Example 4. Consider the third order nonlinear neutral delay difference equation

$$
\begin{aligned}
& \Delta^{3}\left(x_{n}+\frac{1-5 n^{3}}{2+6 n^{3}} x_{n-\tau}\right)+\Delta\left(\frac{2 n^{2}+n-1}{\left(n^{8}+3 n^{6}+2\right)\left(1+x_{3 n-7}^{2}\right)}\right) \\
& \quad+\frac{\sin \left(n^{2} x_{3 n^{2}-2}\right)}{(\sqrt{n}+14)^{22}} \\
& =\frac{(-1)^{n} n^{3}+5 n^{2}+4 n-2}{n^{9}+n^{8}+2 n^{5}+n^{3}+7}, \quad \forall n \geq 9,
\end{aligned}
$$

where $\tau \in \mathbb{N}$ is fixed. Let $n_{0}=9, k=1, b=-5 / 6$, and $\beta=9-\tau$, and let $M$ and $N$ be two positive constants with $M>6 N$ and

$$
\begin{gathered}
b_{n}=\frac{1-5 n^{3}}{2+6 n^{3}}, \quad c_{n}=\frac{(-1)^{n} n^{3}+5 n^{2}+4 n-2}{n^{9}+n^{8}+2 n^{5}+n^{3}+7}, \\
f(n, u)=\frac{\sin \left(n^{2} u\right)}{(\sqrt{n}+14)^{22}}, \\
h(n, u)=\frac{2 n^{2}+n-1}{\left(n^{8}+3 n^{6}+2\right)\left(1+u^{2}\right)}, \\
f_{1 n}=3 n^{2}-2, \quad F_{n}=\left(3 n^{2}-2\right)^{2}, \quad h_{1 n}=3 n-7, \\
H_{n}=(3 n-7)^{2}, \quad P_{n}=Q_{n}=\frac{3}{n^{9}}, \quad R_{n}=W_{n}=\frac{1}{n^{5}}, \\
\quad \forall(n, u) \in \mathbb{N}_{n_{0}} \times \mathbb{R} .
\end{gathered}
$$

That is, (38) and (39) hold. Thus Theorem 5 shows that (86) possesses uncountably many positive solutions in $A(N, M)$. On the other hand, for any $L \in(N,(1 / 6) M)$, there exist $\theta \in(0,1)$ and $T \geq n_{0}+\tau+\beta$ such that the Mann iterative sequence $\left\{x_{m}\right\}_{m \in \mathbb{N}_{0}}=\left\{\left\{x_{m n}\right\}_{n \in \mathbb{N}_{\beta}}\right\}_{m \in \mathbb{N}_{0}}$ generated by (48) converges to a positive solution $z=\left\{z_{n}\right\}_{n \in \mathbb{N}_{\beta}} \in$ $A(N, M)$ of (86) with $\lim _{n \rightarrow \infty} z_{n}=+\infty$ and has the error estimate (20), where $\left\{\alpha_{m}\right\}_{m \in \mathbb{N}_{0}}$ is an arbitrary sequence in $[0,1]$ satisfying $(21)$. 
Example 5. Consider the third order nonlinear neutral delay difference equation

$$
\begin{aligned}
\Delta^{3}( & \left.x_{n}+\left(\frac{\pi}{2}+n \sin \frac{1}{n}\right) x_{n-\tau}\right) \\
+ & \Delta\left(\frac{(-1)^{n(n+1) / 2}}{(n+4)^{8}(n+5)^{3}\left(1+\cos \left(n^{2} x_{2 n+1}\right)\right)}\right) \\
& +\frac{n \sin \left(n x_{n-2}\right)}{2+(n+5)^{16}} \\
= & \frac{(-1)^{n-1} \cos ^{3}\left(n^{2}+1\right)}{n^{16}+\ln n}, \quad \forall n \geq 3,
\end{aligned}
$$

where $\tau \in \mathbb{N}$ is fixed. Let $n_{0}=3, k=1, b=\pi / 2$, and $\beta=\min \{3-\tau, 1\}$, and let $M$ and $N$ be two positive constants with $(1-2 / \pi) M>N$ and

$$
\begin{gathered}
b_{n}=\frac{\pi}{2}+n \sin \frac{1}{n}, \quad c_{n}=\frac{(-1)^{n-1} \cos ^{3}\left(n^{2}+1\right)}{n^{16}+\ln n}, \\
f(n, u)=\frac{n \sin (n u)}{2+(n+5)^{16}}, \\
h(n, u)=\frac{(-1)^{n(n+1) / 2}}{(n+4)^{8}(n+5)^{3}\left(1+\cos \left(n^{2} u\right)\right)}, \\
f_{1 n}=n-2, \quad F_{n}=(n-2)^{2}, \\
h_{1 n}=2 n+1, \quad H_{n}=(2 n+1)^{2}, \\
P_{n}=Q_{n}=\frac{1}{n^{14}}, \quad R_{n}=W_{n}=\frac{2}{n^{9}}, \quad \forall(n, u) \in \mathbb{N}_{n_{0}} \times \mathbb{R} .
\end{gathered}
$$

Clearly, (14), (15), and (61) are satisfied. Note that

$$
\begin{aligned}
\frac{1}{n^{2}} \sum_{u=n}^{\infty} \sum_{s=u}^{\infty} \max \left\{R_{s} H_{s}, W_{s}\right\} \\
\quad=\frac{1}{n^{2}} \sum_{u=n}^{\infty} \sum_{s=u}^{\infty} \max \left\{\frac{2(2 s+1)^{2}}{s^{9}}, \frac{2}{s^{9}}\right\} \\
\quad \leq \frac{18}{n^{2}} \sum_{u=n}^{\infty} \sum_{s=u}^{\infty} \frac{1}{s^{7}} \leq \frac{18}{n^{2}} \sum_{s=n}^{\infty} \frac{1}{s^{6}} \longrightarrow 0 \quad \text { as } n \longrightarrow \infty,
\end{aligned}
$$

which means that

$$
\lim _{n \rightarrow \infty} \frac{1}{n^{2}} \sum_{u=n}^{\infty} \sum_{s=u}^{\infty} \max \left\{R_{s} H_{s}, W_{s}\right\}=0
$$

$$
\begin{aligned}
& \frac{1}{n^{2}} \sum_{u=n}^{\infty} \sum_{s=u}^{\infty} \sum_{t=s}^{\infty} \max \left\{P_{t} F_{t}, Q_{t},\left|c_{t}\right|\right\} \\
& =\frac{1}{n^{2}} \sum_{u=n}^{\infty} \sum_{s=u}^{\infty} \sum_{t=s}^{\infty} \max \left\{\frac{(t-2)^{2}}{t^{14}}, \frac{1}{t^{14}},\right. \\
& \left.\left|\frac{(-1)^{t-1} \cos ^{3}\left(t^{2}+1\right)}{t^{16}+\ln t}\right|\right\} \\
& \leq \frac{1}{n^{2}} \sum_{u=n}^{\infty} \sum_{s=u}^{\infty} \sum_{t=s}^{\infty} \frac{1}{t^{14}} \leq \frac{1}{n^{2}} \sum_{u=n}^{\infty} \sum_{t=u}^{\infty} \frac{1}{t^{13}} \\
& \leq \frac{1}{n^{2}} \sum_{t=n}^{\infty} \frac{1}{t^{12}} \longrightarrow 0 \quad \text { as } n \longrightarrow \infty \text {, }
\end{aligned}
$$

which implies that

$$
\lim _{n \rightarrow \infty} \frac{1}{n^{2}} \sum_{u=n}^{\infty} \sum_{s=u}^{\infty} \sum_{t=s}^{\infty} \max \left\{P_{t} F_{t}, Q_{t},\left|c_{t}\right|\right\}=0
$$

That is, (38) and (39) hold. Consequently Theorem 6 implies that (92) possesses uncountably many positive solutions in $A(N, M)$. On the other hand, for any $L \in((2 / \pi) M+$ $N, M)$, there exist $\theta \in(0,1)$ and $T \geq n_{0}+\tau+$ $\beta$ such that the Mann iterative sequence $\left\{x_{m}\right\}_{m \in \mathbb{N}_{0}}=$ $\left\{\left\{x_{m n}\right\}_{n \in \mathbb{N}_{\beta}}\right\}_{m \in \mathbb{N}_{0}}$ generated by (58) converges to a positive solution $z=\left\{z_{n}\right\}_{n \in \mathbb{N}_{\beta}} \in A(N, M)$ of (92) with $\lim _{n \rightarrow \infty} z_{n}=$ $+\infty$ and has the error estimate (20), where $\left\{\alpha_{m}\right\}_{m \in \mathbb{N}_{0}}$ is an arbitrary sequence in $[0,1]$ satisfying $(21)$.

Example 6. Consider the third order nonlinear neutral delay difference equation

$$
\begin{aligned}
\Delta^{3}\left(x_{n}\right. & \left.-\frac{2 n^{5}+9 n^{2}-1}{n^{5}+3 n^{2}+2} x_{n-\tau}\right)+\Delta\left(\frac{\cos \left((-1)^{n} e^{n}\right)}{(n+7)^{6} \sqrt{1+\left|x_{n-2}\right|}}\right) \\
& +\frac{\sin \left(n^{2} x_{n-1}\right)}{n^{9}+3 n^{5}+2 n^{2}+1} \\
= & \frac{(-1)^{n-1} n^{4}+4 n^{2}+n-1}{n^{11}+6 n^{3}+7 n+2}, \quad \forall n \geq 6,
\end{aligned}
$$

where $\tau \in \mathbb{N}$ is fixed. Let $n_{0}=6, k=1, b=-2$, and $\beta=$ $\min \{6-\tau, 3\}$, and let $M$ and $N$ be two positive constants with $(1 / 2) M>N$ and

$$
b_{n}=-\frac{2 n^{5}+9 n^{2}-1}{n^{5}+3 n^{2}+2}
$$

$$
\begin{aligned}
& c_{n}=\frac{(-1)^{n-1} n^{4}+4 n^{2}+n-1}{n^{11}+6 n^{3}+7 n+2}, \\
& f(n, u)=\frac{\sin \left(n^{2} u\right)}{n^{9}+3 n^{5}+2 n^{2}+1},
\end{aligned}
$$




$$
\begin{gathered}
h(n, u)=\frac{\cos \left((-1)^{n} e^{n}\right)}{(n+7)^{6} \sqrt{1+|u|},} \\
f_{1 n}=n-1, \quad F_{n}=(n-1)^{2}, \\
h_{1 n}=n-2, \quad H_{n}=(n-2)^{2}, \\
P_{n}=Q_{n}=\frac{1}{n^{7}}, \quad R_{n}=W_{n}=\frac{1}{n^{6}}, \\
\forall(n, u) \in \mathbb{N}_{n_{0}} \times \mathbb{R} .
\end{gathered}
$$

Obviously, (14), (15), and (64) are satisfied. Note that

$$
\begin{aligned}
\frac{1}{n^{2}} & \sum_{u=n}^{\infty} \sum_{s=u}^{\infty} \max \left\{R_{s} H_{s}, W_{s}\right\} \\
& =\frac{1}{n^{2}} \sum_{u=n}^{\infty} \sum_{s=u}^{\infty} \max \left\{\frac{(s-2)^{2}}{s^{6}}, \frac{1}{s^{6}}\right\} \\
& \leq \frac{1}{n^{2}} \sum_{u=n}^{\infty} \sum_{s=u}^{\infty} \frac{1}{s^{4}} \leq \frac{1}{n^{2}} \sum_{s=n}^{\infty} \frac{1}{s^{3}} \longrightarrow 0 \quad \text { as } n \longrightarrow \infty,
\end{aligned}
$$

which means that

$$
\lim _{n \rightarrow \infty} \frac{1}{n^{2}} \sum_{u=n}^{\infty} \sum_{s=u}^{\infty} \max \left\{R_{s} H_{s}, W_{s}\right\}=0 .
$$

It is clear that

$$
\begin{aligned}
& \frac{1}{n^{2}} \sum_{u=n}^{\infty} \sum_{s=u}^{\infty} \sum_{t=s}^{\infty} \max \left\{P_{t} F_{t}, Q_{t},\left|c_{t}\right|\right\} \\
& =\frac{1}{n^{2}} \sum_{u=n}^{\infty} \sum_{s=u}^{\infty} \sum_{t=s}^{\infty} \max \left\{\frac{(t-1)^{2}}{t^{7}}, \frac{1}{t^{7}},\right. \\
& \leq \frac{1}{n^{2}} \sum_{u=n}^{\infty} \sum_{s=u}^{\infty} \sum_{t=s}^{\infty} \frac{1}{t^{5}} \leq \frac{1}{n^{2}} \sum_{u=n}^{\infty} \sum_{t=u}^{\infty} \frac{1}{t^{4}}+7 t^{2}+t-1 \\
& \quad \leq \frac{1}{n^{2}} \sum_{t=n}^{\infty} \frac{1}{t^{3}} \longrightarrow 0 \quad \text { as } n \longrightarrow \infty,
\end{aligned}
$$

which implies that

$$
\lim _{n \rightarrow \infty} \frac{1}{n^{2}} \sum_{u=n}^{\infty} \sum_{s=u}^{\infty} \sum_{t=s}^{\infty} \max \left\{P_{t} F_{t}, Q_{t},\left|c_{t}\right|\right\}=0
$$

That is, (38) and (39) hold. Consequently Theorem 7 implies that (97) possesses uncountably many positive solutions in $A(N, M)$. On the other hand, for any $L \in(-M / 2,-N)$, there exist $\theta \in(0,1)$ and $T \geq n_{0}+\tau+\beta$ such that the Mann iterative sequence $\left\{x_{m}\right\}_{m \in \mathbb{N}_{0}}=\left\{\left\{x_{m n}\right\}_{n \in \mathbb{N}_{\beta}}\right\}_{m \in \mathbb{N}_{0}}$ generated by (65) converges to a positive solution $z=$ $\left\{z_{n}\right\}_{n \in \mathbb{N}_{\beta}} \in A(N, M)$ of (97) with $\lim _{n \rightarrow \infty} z_{n}=+\infty$ and has the error estimate (20), where $\left\{\alpha_{m}\right\}_{m \in \mathbb{N}_{0}}$ is an arbitrary sequence in $[0,1]$ satisfying $(21)$.

\section{Conflict of Interests}

The authors declare that there is no conflict of interests regarding the publication of this paper.

\section{Acknowledgment}

This research was supported by the Science Research Foundation of Educational Department of Liaoning Province (L2012380).

\section{References}

[1] M. H. Abu-Risha, "Oscillation of second-order linear difference equations," Applied Mathematics Letters, vol. 13, no. 1, pp. 129$135,2000$.

[2] R. P. Agarwal, Difference Equations and Inequalities, Marcel Dekker, New York, NY, USA, 2nd edition, 2000.

[3] R. P. Agarwal and J. Henderson, "Positive solutions and nonlinear eigenvalue problems for third-order difference equations," Computers \& Mathematics with Applications, vol. 36, no. 10-12, pp. 347-355, 1998.

[4] A. Andruch-Sobiło and M. Migda, "On the oscillation of solutions of third order linear difference equations of neutral type," Mathematica Bohemica, vol. 130, no. 1, pp. 19-33, 2005.

[5] Z. Došlá and A. Kobza, "Global asymptotic properties of thirdorder difference equations," Computers \& Mathematics with Applications, vol. 48, no. 1-2, pp. 191-200, 2004.

[6] S. R. Grace and G. G. Hamedani, "On the oscillation of certain neutral difference equations," Mathematica Bohemica, vol. 125, no. 3, pp. 307-321, 2000.

[7] J. Cheng, "Existence of a nonoscillatory solution of a secondorder linear neutral difference equation," Applied Mathematics Letters, vol. 20, no. 8, pp. 892-899, 2007.

[8] L. Kong, Q. Kong, and B. Zhang, "Positive solutions of boundary value problems for third-order functional difference equations," Computers \& Mathematics with Applications, vol. 44, no. 3-4, pp. 481-489, 2002.

[9] I. Y. Karaca, "Discrete third-order three-point boundary value problem," Journal of Computational and Applied Mathematics, vol. 205, no. 1, pp. 458-468, 2007.

[10] W.-T. Li and J. P. Sun, "Existence of positive solutions of BVPs for third-order discrete nonlinear difference systems," Applied Mathematics and Computation, vol. 157, no. 1, pp. 53-64, 2004.

[11] W.-T. Li and J.-P. Sun, "Multiple positive solutions of BVPs for third-order discrete difference systems," Applied Mathematics and Computation, vol. 149, no. 2, pp. 389-398, 2004.

[12] Z. Liu, M. Jia, S. M. Kang, and Y. C. Kwun, "Bounded positive solutions for a third order discrete equation," Abstract and Applied Analysis, vol. 2012, Article ID 237036, 12 pages, 2012.

[13] Z. Liu, S. M. Kang, and J. S. Ume, "Existence of uncountably many bounded nonoscillatory solutions and their iterative approximations for second order nonlinear neutral delay difference equations," Applied Mathematics and Computation, vol. 213, no. 2, pp. 554-576, 2009.

[14] Z. Liu, Y. Xu, and S. M. Kang, "Bounded oscillation criteria for certain third order nonlinear difference equations with several delays and advances," Computers \& Mathematics with Applications, vol. 61, no. 4, pp. 1145-1161, 2011.

[15] M. Migda and J. Migda, "Asymptotic properties of solutions of second-order neutral difference equations," Nonlinear Analysis: 
Theory, Methods and Applications, vol. 63, no. 5-7, pp. e789e799, 2005.

[16] N. Parhi, "Non-oscillation of solutions of difference equations of third order," Computers \& Mathematics with Applications, vol. 62, no. 10, pp. 3812-3820, 2011.

[17] N. Parhi and A. Panda, "Nonoscillation and oscillation of solutions of a class of third order difference equations," Journal of Mathematical Analysis and Applications, vol. 336, no. 1, pp. 213-223, 2007.

[18] S. H. Saker, "New oscillation criteria for second-order nonlinear neutral delay difference equations," Applied Mathematics and Computation, vol. 142, no. 1, pp. 99-111, 2003.

[19] S. H. Saker, "Oscillation of third-order difference equations," Portugaliae Mathematica, vol. 61, no. 3, pp. 249-257, 2004.

[20] S. Stević, "On a third-order system of difference equations," Applied Mathematics and Computation, vol. 218, no. 14, pp. 7649-7654, 2012.

[21] X. H. Tang, "Bounded oscillation of second-order delay difference equations of unstable type," Computers \& Mathematics with Applications, vol. 44, no. 8-9, pp. 1147-1156, 2002.

[22] J. Yan and B. Liu, "Asymptotic behavior of a nonlinear delay difference equation," Applied Mathematics Letters, vol. 8, no. 6, pp. 1-5, 1995.

[23] Z. G. Zhang and Q. L. Li, "Oscillation theorems for secondorder advanced functional difference equations," Computers \& Mathematics with Applications, vol. 36, no. 6, pp. 11-18, 1998. 




Advances in

Operations Research

mansans



The Scientific World Journal
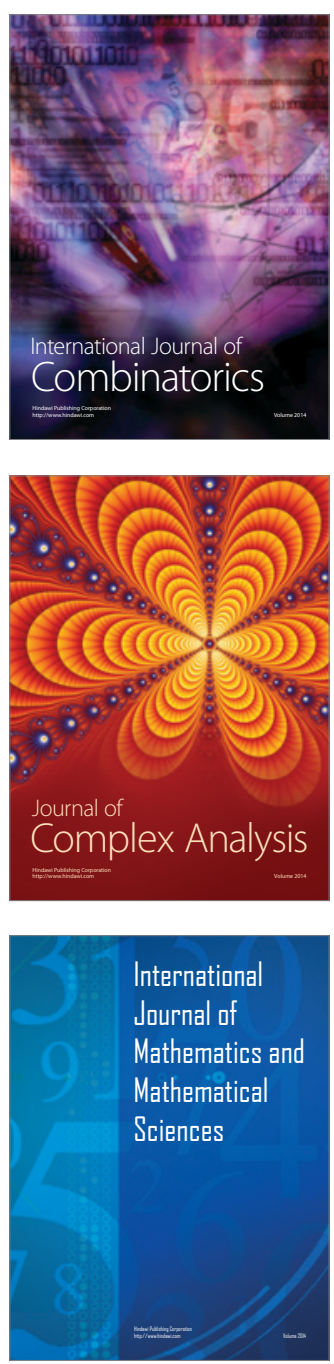
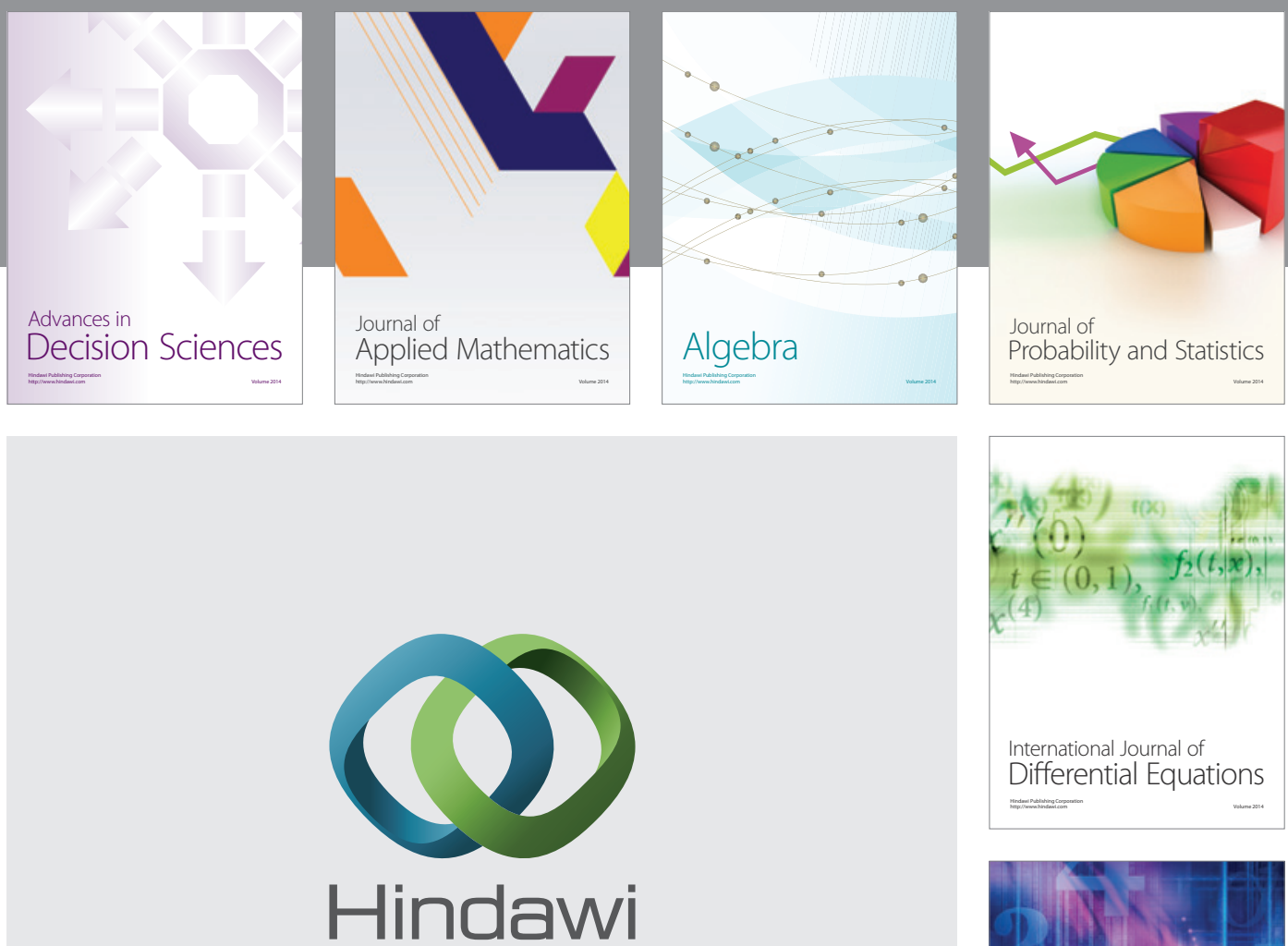

Submit your manuscripts at http://www.hindawi.com
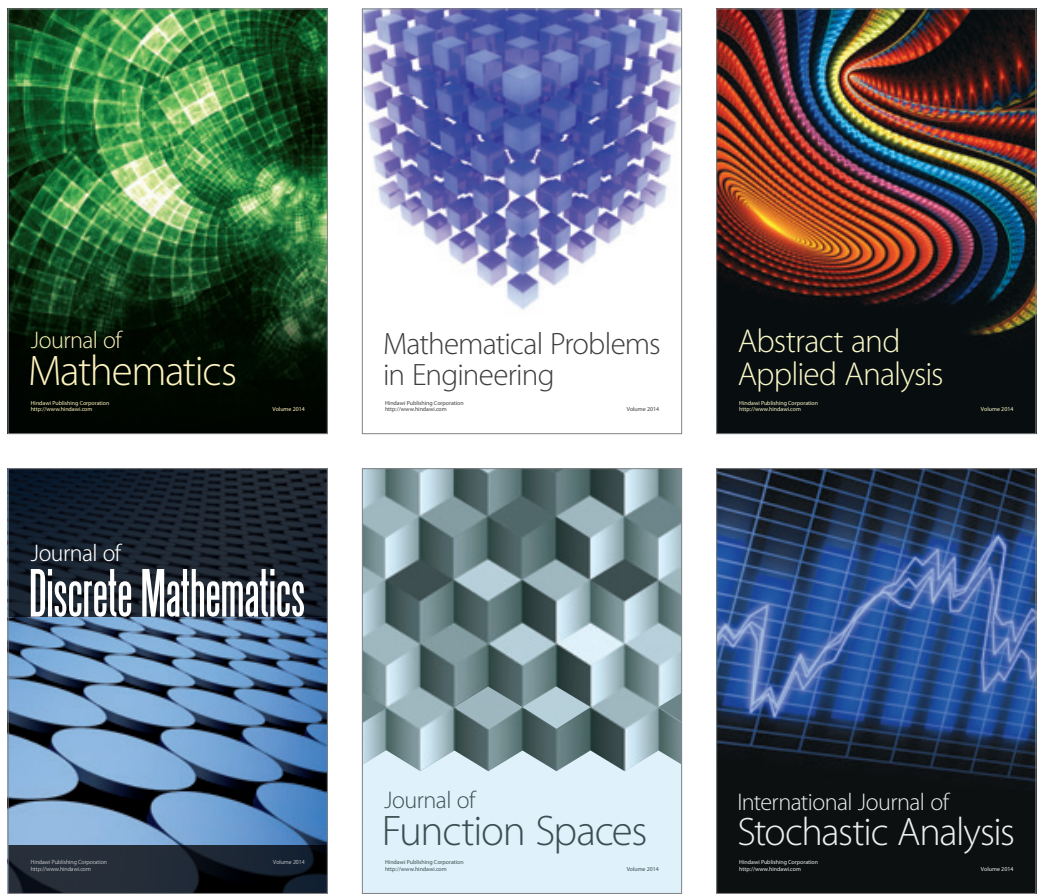

Journal of

Function Spaces



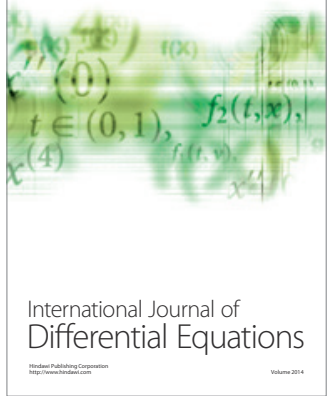
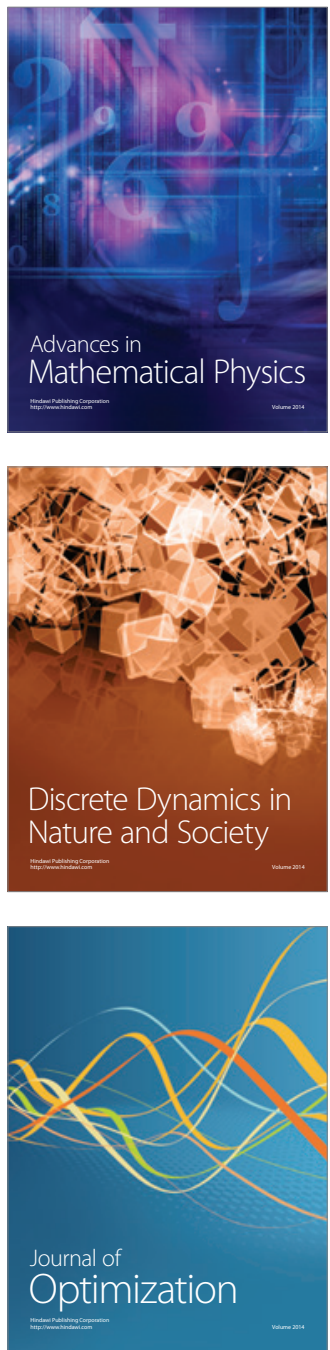